\title{
Improved Hartree-Fock resummations and spontaneous symmetry breaking
}

\author{
Andrea Sartirana* and Claudio Destri ${ }^{\dagger}$ \\ Dipartimento di Fisica, dell'Università degli studi di Milano-Bicocca, Milano, Italy \\ and INFN, Sezione di Milano, piazza della Scienza 3, I-20126 Milano, Italy
}

(Received 7 September 2005; published 18 January 2006)

\begin{abstract}
The standard Hartree-Fock approximation of the $O(N)$-invariant $\phi^{4}$ model suffers from serious renormalization problems. In addition, when the symmetry is spontaneously broken, another shortcoming appears in relation to the Goldstone bosons: they fail to be massless in the intermediate states. In this work, within the framework of out-of-equilibrium Quantum Field Theory, we propose a class of systematic improvements of the Hartree-Fock resummation which overcomes all the above mentioned difficulties while ensuring also exact Renormalization-Group invariance to one loop.
\end{abstract}

DOI: 10.1103/PhysRevD.73.025012

PACS numbers: 11.10.Ef, 11.10.Gh, 11.15.Pg

\section{INTRODUCTION, SUMMARY, AND OUTLOOK}

The research in out-of-equilibrium dynamics of quantum fields has received, in recent years, a great impulse by cosmology as well as by particle and condensed matter physics. Indeed, a first-principle theoretical treatment of many important phenomena, such as the reheating of the Universe after inflation or the thermalization of the quark gluon plasma in the ultrarelativistic heavy-ion colliders (RHIC, LHC), requires for a good qualitative and quantitative understanding of the late time and strongly coupled evolution of quantum field systems.

In particular, this necessity has encouraged the study of nonperturbative approaches to Quantum Field Theory (QFT) that could provide all-orders partial resummations of Feynman diagrams $[1,2]$. In fact standard perturbation theory does not yield satisfactory results, except for very short times, when nonequilibrium conditions are involved.

Mean-field approximations such as leading-order large- $N$ expansion [3-6] and Hartree, or Hartree-Fock (HF) variational method [7-12] are the simplest and most studied [13-18] resummation schemes. Their main features are well known: they do provide a backreaction term on the evolution of quantum fluctuations that stabilize dynamics after parametric amplifications or spinodal instabilities. On the other hand they fail to reproduce important properties of late time dynamics such as thermalization as well as thermodynamical properties such the order of the unbroken/broken symmetry phase transition. A more powerful tool is the 2PI (or 2PPI) effective action $[19,20]$ whose expansions at two (or more) loops order or at next-to-leading order in $1 / N$ provide resummations that go beyond mean-field approaches [21-24] and yield, indeed, approximate numerical thermalization at strong coupling.

More formal aspects of resummed approximations, such as their renormalization properties, have been studied as well [25-27]. Recently a systematic method has been put

\footnotetext{
*Email: andrea.sartirana@mib.infn.it

${ }^{\dagger}$ Email: claudio.destri@mib.infn.it
}

forward [28-32] that removes divergences in the $\Phi$-derivable approximations by applying a BogoliubovParasiuk-Hepp-Zimmerman (BPHZ) subtraction procedure to diagrams with resummed propagators.

In [33] we considered the simple HF approximation of the $O(N) \phi^{4}$ model in the unbroken symmetry phase. It is known that it cannot be consistently renormalized by the usual renormalization of bare coupling and mass $[8,25,26]$. We showed that this nonrenormalizability is due to the absence of leading logarithmically divergent contributions coming from diagrams which do not have a "daisy" topology and therefore are not present in the standard HF resummation. The inclusion of these contribution together with suitably chosen finite parts led us to the definition of a renormalized and Renormalization-Group invariant version of HF equations.

In the present paper we further develop this analysis, making it more systematic and transparent, and then apply it to the subtler case of spontaneously broken $O(N)$ symmetry. In this case, together with the shortcomings as far as pure renormalizability is concerned, the HF approximation shows an unphysical nongapless behavior. Namely, the effective resummed propagators of the Goldstone bosons, as defined by the HF equation of motion, are not massless. This is a well-known problem which has been cured by defining suitably modified approximations [34,35]. In this paper we define a systematic improvement of the standard HF approach which ensures gaplessness together with renormalizability and RG-invariance. In practice we include all leading logarithmically divergent contributions needed to render finite the resummed propagator masses (which are $\log \Lambda$ dependent in standard HF) while choosing the finite parts in such a way to have massless transverse degrees of freedom.

More in detail, we proceed as follows. First, by analyzing the diagrammatic resummation performed by the HF approximation, we identify the missing leading logarithmically divergent contributions that cause the nonrenormalizability. As will be apparent below, this analysis closely parallels that in [33] since, after all, the UV behavior of the 
theory is the same regardless of the breaking of the $O(N)$ symmetry. Then we construct our modified HF approximation, including the missing contributions, while choosing the corresponding finite parts by means of the following recipe

(a) determine the general features of the HF approach that must be shared by the modified approximation, such as mean-field structure, $O(N)$ Ward identities, leading-log structure and more, and parameterize the class of resummations having such properties.

(b) identify amid this family of approximations the one(s) with suitable properties of renormalizability, RG-invariance, gaplessness and infrared finiteness.

One important difference from [33] is that, when the $O(N)$ symmetry is spontaneously broken, the above procedure does not lead to a unique result except for the $N=1$ case ( $\mathbb{Z}_{2}$ symmetry). When $N>1$ we find a whole class of approximations, corresponding to different choices of finite parts, that share the required characteristics. Like in [33] the improved equations have, by construction, a meanfield structure but, unlike the standard ones, are nonlocal in space and time.

In Sec. II we review some general concepts. We introduce the closed time path (CTP) formulation of out-ofequilibrium problems and define the HF approximation as resummation of bubble diagrams recalling the corresponding, well-known, equations of motion [see Eqs. (2.4)]. Some important general features of the mean-field approximations are pointed out.

In Sec. III we study, as an illuminating example, the case of spontaneously broken $\mathbb{Z}_{2}$ symmetry. Subsection III A is dedicated to a general analysis of the approximation. In Subsection III B we apply the standard renormalization procedure and point out its shortcomings. In Subsection III C we define our modified HF approximation which is renormalizable and RG-invariant. As we already said in this case we are led to a unique result [see Eqs. (3.19) and Eqs. (3.22)].

In Sec. IV we generalize to $N>1$, that is to a continuous $O(N)$ spontaneous symmetry breaking, by first analyzing some general features [Subsection IVA] of the standard HF approximation and its renormalization properties [Subsection IV B] and then defining our modified approximation by the general recipe given above [Subsection IV C]. The final results will be a set of constraints that should be satisfied by renormalizable and RGinvariant Hartree-Fock-like approximations together with two examples [see Eqs. (4.27) and Eqs. (4.28)].

There are several possible developments along the lines of this work. First of all it would be interesting to study other properties of the different improved approximations that we have constructed in the $N>1$ case and, hopefully, identify further constraints that would allow to single out a unique result. Secondly, a numerical study of the modified HF field equations found in this paper and in [33] would be needed to investigate how the space-time nonlocalities affect the time evolution as compared to the standard HF approximation, which is known to fail even qualitatively at late times.

The aim of this paper is to develop the formalism and further investigations are needed to understand whether the improvement of the HF resummation field-theoretic properties reflects in a physically improved behavior. Moreover the work on the HF approximation must be regarded as preliminary to the application of similar analysis to more sophisticated resummation methods or to more phenomenologically interesting theories. For example a challenging task would be the extension of our approach to the full two-loop 2PI effective action, through the inclusion of the nonlocal sunset diagram which is absent by definition in any mean-field approximation. In fact one should expect that, even if such an inclusion allows to recover renormalizability as compared to the conventional HF approximation, the two-loop 2PI self-consistent equations still lack RG invariance with the two-loop beta function, since the 2PI effective action does not contain all the diagrams which contribute to the next-to-leading ultraviolet divergences.

\section{GENERALITIES}

Out-of-equilibrium QFT is defined as the study of realtime dynamics of quantum fields and provides the general setup for the calculation of in-in matrix elements (i.e. expectation values of operators on a given initial state). In this section we briefly review some generalities about nonequilibrium QFT, define the Hartree-Fock approximation, and point out some useful properties. We deal with the scalar field theory in $3+1$ dimensions with quartic interaction and spontaneously broken $O(N)$ symmetry,

$$
\begin{aligned}
S[\varphi]= & \int d^{4} x\left\{\frac{1}{2} \partial_{\mu} \varphi_{i}(x) \partial^{\mu} \varphi_{i}(x)-\frac{m^{2}}{2} \varphi_{i}(x) \varphi_{i}(x)-\frac{\lambda}{4 !}\right. \\
& \left.\times\left[\varphi_{i}(x) \varphi_{i}(x)\right]^{2}\right\} .
\end{aligned}
$$

$m^{2}$ and $\lambda$ are the (negative) squared mass and the coupling constant, respectively.

In QFT, the general approach to nonequilibrium dynamics was developed by Keldish and Schwinger and it allows to use standard functional methods (see $[19,20,36,37]$ ) by introducing path integrals on a CTP going from $t=0$ to $t=+\infty$ and back. Field integration variables for the path integral forward and backward in time are denoted as $(+)$ and (-)-components, respectively. Given an initial state $\Psi$ one writes down the functional integral for the connected Green functions generator

$$
\begin{aligned}
e^{i \mathcal{W}\left[j_{+}, j_{-}\right]}= & \int \mathcal{D} \varphi_{+} \mathcal{D} \varphi_{-} \Psi\left[\varphi_{+}\right] \bar{\Psi}\left[\varphi_{-}\right] \\
& \times e^{i S\left[\varphi_{+}\right]-i S\left[\varphi_{-}\right]+i\left\langle j_{+} \mid \varphi_{+}\right\rangle-i\left\langle j_{-} \mid \varphi_{-}\right\rangle},
\end{aligned}
$$

where we have used an obvious short-hand notation for the 
current terms. Integration in Eq. (2.1) is on trajectories from $t=0$ to $t=+\infty$ (with the condition $\varphi_{+}=\varphi_{-}$at $t=+\infty)$ and $\varphi_{ \pm}$in the wave functional is the $t=0$ section of $\varphi_{+}$. The effective action $\Gamma_{1 \mathrm{PI}}$ is the Legendre transform of $\mathcal{W}$ from the currents $j_{ \pm}$to the fields $\phi_{ \pm}$. The equation of motion for the background field $\phi(x)=$ $\langle\Psi|\varphi(x)| \Psi\rangle$ then reads

$$
\left.\frac{\delta \Gamma_{1 \mathrm{PI}}}{\delta \phi_{+}(x)}\right|_{\phi_{+}=\phi_{-}=\phi}=0 .
$$

In our present discussion we consider an initial wave functional having the following Gaussian form

$$
\begin{aligned}
\Psi[\varphi]= & \mathcal{N} \exp \{i\langle\dot{\phi}(0) \mid \varphi-\phi(0)\rangle \\
& \left.-\left\langle\varphi-\phi(0)\left|\left[\frac{1}{4} G^{-1}+i S\right]\right| \varphi-\phi(0)\right\rangle\right\}
\end{aligned}
$$

whose free parameters are the $t=0$ background field $\phi(x, 0)$, the $t=0$ background momentum $\dot{\phi}(\boldsymbol{x}, 0)$, the real symmetric positive kernel $G_{i j}(\boldsymbol{x}, \boldsymbol{y})$, and the real symmetric kernel $S_{i j}(\boldsymbol{x}, \boldsymbol{y})$.

The perturbative diagrammatic expansion in the CTP formalism proceeds as in vacuum QFT. However, sensible results in out-of-equilibrium contexts can be obtained only going beyond plain perturbation and performing (partial) resummations to all orders in the coupling constant. A very successful tool in this sense is the 2PI effective action (see $[19,20])$. It is defined as the double Legendre transform of the $\mathcal{W}$ generating functional with respect to the usual current one-point $j_{ \pm}$and to the two-points current $K_{\alpha \beta}(x, y)$ quadratically coupled to fields. It yields two equations of motions

$$
\left.\frac{\delta \Gamma_{2 \mathrm{PI}}}{\delta \phi_{\alpha}(x)}\right|_{*}=0,\left.\quad \frac{\delta \Gamma_{2 \mathrm{PI}}}{\delta G_{\alpha \beta}(x, y)}\right|_{*}=0 .
$$

Here the notation $\left.\right|_{*}$ indicates that, by their physical meaning, the (士)-component fields and propagators have to satisfy, on the solutions of motion, the following relations

$$
\begin{aligned}
\phi_{-}(x) & =\phi_{-}(x)=\phi(x), \\
G_{F}(x, y) & =G_{+-}(y, x) \theta\left(x_{0}-y_{0}\right)+G_{+-}(x, y) \theta\left(y_{0}-x_{0}\right), \\
G_{\bar{F}}(x, y) & =G_{+-}(x, y) \theta\left(x_{0}-y_{0}\right)+G_{+-}(y, x) \theta\left(y_{0}-x_{0}\right) .
\end{aligned}
$$

Hence the system Eq. (2.3) reduces to two coupled equations for $\phi$ and $G_{+-}$only. The $\Gamma_{2 \text { PI }}$ at a certain perturbative loop order corresponds (i.e. gives the same equations) to a resummed diagrammatic approximation of the 1PI effective action. In the present scalar theory $\Gamma_{2 \mathrm{PI}}$ has the general form

$$
\begin{aligned}
\Gamma_{2 \mathrm{PI}}[\phi, G]= & S[\phi]+\frac{i}{2} \operatorname{Tr}[\log G]+\frac{i}{2} \operatorname{Tr}\left[G_{0}^{-1} G\right] \\
& +\Gamma_{2}[\phi, G],
\end{aligned}
$$

where $S$ is the complete classical action of the double time path (i.e. $S=S_{+}-S_{-}$). Traces are taken over all indices $i, \alpha$, and $x . G_{0}^{-1}$ is the second derivative of the action in a $\phi$ background, $\Gamma_{2}$ is the sum of all vacuum 2PI diagrams with $G$ propagators and vertices defined by the classical action in a $\phi$ background. To two-loops level the diagrams contributing to the $\Gamma_{2}$ are the " 8 " and "sunset" diagrams. The Hartree-Fock approximation is obtained considering only the 8 graph. In the 1PI framework this corresponds to a resummation of all vacuum 1PI diagrams with daisy and superdaisy topologies. The corresponding equations of motion are conveniently expressed in terms of mode functions $u_{k a}(\boldsymbol{k}$ is the wave vector and $a$ the $O(N)$ polarization)

$$
\begin{array}{r}
\left\{\left[\square+m_{0}^{2}+\frac{1}{6} \lambda_{0} \phi_{k}(x) \phi_{k}(x)\right] \delta_{i j}\right. \\
\left.+\frac{1}{2} \lambda_{0} \tau_{i j k m} G_{k m}(x, x)\right\} \phi_{j}(x)=0, \\
\left\{\left(\square+m_{0}^{2}\right) \delta_{i j}+\frac{1}{2} \lambda_{0} \tau_{i j k m}\left[\phi_{k}(x) \phi_{m}(x)\right.\right. \\
\left.\left.+G_{k m}(x, x)\right]\right\} u_{k, a j}=0,
\end{array}
$$

where the cutoffed correlation $G$ and the tensor $\tau$ are defined

$$
\begin{aligned}
G_{i j}(x, y) & =\operatorname{Re} \int_{|p|^{2}<\Lambda} \frac{d^{3} k}{(2 \pi)^{3}} u_{k, a i}(x) \bar{u}_{k, a j}(y), \\
\tau_{i j k m} & =\frac{1}{3}\left(\delta_{i j} \delta_{k m}+\delta_{i k} \delta_{j m}+\delta_{i m} \delta_{j k}\right) .
\end{aligned}
$$

The $t=0$ mode functions are

$$
\begin{aligned}
& u_{\boldsymbol{k}, a i}(\boldsymbol{x}, 0)=\left[\tilde{\mathcal{G}}(\boldsymbol{k})^{1 / 2}\right]_{a i} e^{i \boldsymbol{k} \cdot \boldsymbol{x}}, \\
& \dot{u}_{\boldsymbol{k}, a i}(\boldsymbol{x}, 0)=\left[-\frac{i}{2} \tilde{\mathcal{G}}(\boldsymbol{k})^{-1}+2 \tilde{S}(\boldsymbol{k})\right]_{i j} u_{\boldsymbol{k}, a j}(\boldsymbol{x}, 0) .
\end{aligned}
$$

Notice that, for simplicity, we have supposed that the initial $(t=0)$ kernels are translationally invariant.

We conclude this section by introducing the physical representation (see [37]) of the CTP formalism. It is defined by the field redefinitions

$$
\begin{aligned}
\phi_{\Delta}=\phi_{+}-\phi_{-}, \phi_{c} & =\frac{1}{2}\left(\phi_{+}+\phi_{-}\right) \\
\left.\longrightarrow \quad \frac{\delta \Gamma}{\delta \phi_{\Delta}}\right|_{\phi_{\Delta}=0, \phi_{c}=\phi} & =0 .
\end{aligned}
$$

The equations of motion written above are obtained using the property that variations of $\Gamma$ with respect to $\phi_{c}$ fields alone vanish when $\phi_{\Delta}=0$. Notice that $2 n$-legs vertex functions with one $\phi_{\Delta}$ leg and $2 n-1 \phi_{c}$ legs are the only ones contributing to these equations of motion. One can show that these vertices are time ordered in such a way to ensure causality. As stated above, the HF approximation consists in the resummation of diagrams with daisy and superdaisy topologies thus it is easy to verify the following general structure of the effective action

$$
\Gamma_{\mathrm{HF}}\left[\phi_{\Delta}, \phi_{c}\right]=-\left\langle\phi_{\Delta}|\square| \phi_{c}\right\rangle-\mathcal{F}[\xi, \chi, \eta],
$$

where $\mathcal{F}$ is a functional of the following composite matrix fields 


$$
\begin{gathered}
\xi_{i j}(x)=\phi_{c, i}(x) \phi_{c, j}(x), \quad \chi_{i j}(x)=\phi_{c, i}(x) \phi_{\Delta, j}(x), \\
\eta_{i j}(x)=\phi_{\Delta, i}(x) \phi_{\Delta, j}(x) .
\end{gathered}
$$

Then the equation of motion in the HF approximation takes the form

$$
\begin{aligned}
\left.\frac{\delta \Gamma}{\delta \phi_{\Delta}}\right|_{\phi_{\Delta}=0, \phi_{c}=\phi} & =\left\{\delta_{i j}+2 \mathcal{F}_{i j}^{\prime}[\xi]\right\} \phi_{j}=0, \\
\mathcal{F}^{\prime}[\xi]_{i j}(x) & =\left.\frac{1}{2} \frac{\delta \mathcal{F}}{\delta \chi_{i j}(x)}\right|_{\chi=\eta=0} .
\end{aligned}
$$

We will consider this as a general form for mean-field-type background field equations and it will be that base for our definition of a modified HF approximation.

\section{THE CASE $N=1$}

We begin by considering a single scalar field theory with spontaneously broken $\mathbb{Z}_{2}$ symmetry. The study of this simpler case provides valuable insight into the general features of the diagrammatic resummation performed by the HF approximation. In particular, this allows to understand the origin of the HF shortcomings with respect to renormalizability and RG invariance and to determine the general recipe for the definition of a modified renormalizable and RG-invariant mean-field approximation. Many of the results of this section will hold true also in the more general case of a theory with $N$ scalar fields.

\section{A. Analysis of the HF approximation}

We begin by the HF equations of motion [see Eqs. (2.4)] reduced to the $N=1$ case. So far these are still the equations of the bare theory written in terms of $\lambda_{0}, m_{0}^{2}$, and explicitly dependent on the sharp cutoff $\Lambda$. The phase with spontaneously broken symmetry is defined by assuming the existence of a static homogeneous vacuum solution with nonzero background field $\phi(x)=v$. The corresponding mode functions $u^{(\mathrm{vac})}$ have the following plane wave form

$$
u_{\boldsymbol{k}}^{(\mathrm{vac})}(x)=\frac{1}{\sqrt{2 \omega_{\boldsymbol{k}}}} e^{i\left(\boldsymbol{k} \cdot \boldsymbol{x}-\omega_{\boldsymbol{k}} t\right)}, \quad \omega_{\boldsymbol{k}}^{2}=\boldsymbol{k}^{2}+m^{2},
$$

where $m^{2}=\frac{1}{3} \lambda_{0} v^{2}$ and the vacuum expectation value $v$ of the background should satisfy the gap equation

$$
0=m_{0}^{2}+\frac{1}{6} \lambda_{0} v^{2}+\frac{1}{2} \lambda_{0} \int_{p^{2}<\Lambda} \frac{d^{3} p}{(2 \pi)^{3}} \frac{1}{2 \omega_{k}} .
$$

The values of bare parameters for which this equation admits a nonzero solution $v$ are those corresponding to spontaneously broken symmetry and are those we are considering here.
We now introduce the mean field $\mathcal{V}$ according to

$$
\begin{aligned}
\mathcal{V}(x)= & m_{0}^{2}+\frac{1}{6} \lambda_{0} v^{2}+\frac{1}{2} \lambda_{0} \Delta \xi(x) \\
& +\frac{1}{2} \lambda_{0} \int_{p^{2}<\Lambda} \frac{d^{3} p}{(2 \pi)^{3}}\left|u_{p}(x)\right|^{2},
\end{aligned}
$$

where $\Delta \xi=\xi-v^{2}$ (we recall that $\xi(x)=\phi^{2}(x)$ ). Notice that $V=0$ on the static solution $\phi=v$ and $u=u^{\text {(vac) }}$. This definition allows us to rewrite the equations of motion in the form

$$
\begin{aligned}
\left\{\square-\frac{1}{3} \lambda_{0} \Delta \xi(x)+\mathcal{V}(x)\right\} \phi(x) & =0, \\
\left\{\square+m^{2}+\mathcal{V}(x)\right\} u_{k}(x) & =0 .
\end{aligned}
$$

Comparing Eq. (2.6) with the first of Eqs. (3.4), we read out

$$
\mathcal{F}^{\prime}[\xi]=\frac{1}{2} \mathcal{V}-\frac{1}{6} \lambda_{0} \Delta \xi .
$$

Notice that $\mathcal{V}$ is regarded here as a functional of $\xi=\phi^{2}$. The implicit dependence on $\xi$ is determined by solving the second equation in (3.4) with a generic background. To obtain a self-consistent equation for the functional $\mathcal{V}[\xi]$ from the HF equations of motion we cast the equation of motion for the mode functions into a convenient integral form

$$
u_{k}(x)=u_{k}^{(0)}(x)+\int d^{4} y G_{R}^{(0)}(x-y) \mathcal{V}(y) u_{k}(y),
$$

where the free mode functions $u_{k}^{(0)}$ defined as solutions of the free equation with mass $m^{2}=\frac{1}{3} \lambda_{0} v^{2}$ and with the same initial conditions of the exact mode functions, while the free retarded (and advanced) Green functions reads

$$
\begin{aligned}
G_{R}^{(0)}(x-y) & =G_{A}^{(0)}(y-x) \\
& =\int \frac{d^{4} p}{(2 \pi)^{4}} \frac{1}{p^{2}-m^{2}+i \epsilon p_{0}} e^{-i p(x-y)} .
\end{aligned}
$$

In this equation, and everywhere else from now on, all fields ( $\phi$, mode functions, $\mathcal{F}^{\prime}, \mathcal{V}$, etc.) are to be thought as defined only for positive times (initial conditions are at the limit point $t=0^{+}$) and all time integrations are restricted to positive values, as appropriate in an initial value problem. Moreover it is convenient to introduce a more compact operator notation with implicit space-time integration, that is

$$
u_{\boldsymbol{k}}=u_{\boldsymbol{k}}^{(0)}+G_{R}^{(0)} \hat{V} u_{k},
$$

where the caret ${ }^{\wedge}$ turns a vector of the functional space into a multiplication operator

$$
\hat{:} \boldsymbol{v}(x) \rightarrow \hat{v}(x, y)=v(x) \delta^{(4)}(x-y) .
$$

From now on we shall use this notation throughout the paper. We can formally solve Eq. (3.7) and obtain an expression for the correlation [see Eq. (2.5)] 


$$
\begin{aligned}
& u_{k}=\left[\mathbf{1}-G_{R}^{(0)} \hat{\mathcal{V}}\right]^{-1} u_{k}^{(0)}, \\
& G=G[\mathcal{V}]=\left[\mathbf{1}-G_{R}^{(0)} \hat{\mathcal{V}}\right]^{-1} G^{(0)}\left[\mathbf{1}-\hat{\mathcal{V}} G_{A}^{(0)}\right]^{-1},
\end{aligned}
$$

where 1 stands for the space-time delta function and $G^{(0)}$ is the correlation in Eq. (2.5) evaluated in the free mode functions. Substituting into the definition of $\mathcal{V}$ in Eq. (3.3) we obtain the sought self-consistent equation

$$
\begin{aligned}
\mathcal{V} & =m_{0}^{2}+\frac{1}{6} \lambda_{0} v^{2}+\frac{1}{2} \lambda_{0}(\Delta \xi+I[\mathcal{V}]), \\
I[\mathcal{V}](x) & =G[\mathcal{V}](x, x) .
\end{aligned}
$$

Notice that Eq. (3.9) depends parametrically on the initial kernels (i.e. the mode functions initial conditions) through the explicit form of $G^{(0)}$. Now, before going any further in the discussion, we fix a particular choice for these kernels by considering equilibrium initial conditions for the mode functions. By this choice follows that the free mode functions coincide with the vacuum mode functions $u^{\text {(vac) }}$ at every time (by the self-consistent equation we have $\mathcal{V}=0$ at the point $\left.\xi(x)=v^{2}\right)$ the free correlation function turns to be translationally invariant in space-time. However let us stress that, in spite of this choice, we are still considering an out-of-equilibrium problem since we allow for generic initial conditions for the background field.

At this point some observations on the integral term $I$ are in order.

(1) $I$ depends on the free retarded (and advanced) Green function and on the free correlation function. We can see that their definition corresponds to the resummation of all tadpole corrections and they play the role of internal dressed propagators.

(2) By expanding $I$ in powers of $\mathcal{V}$ we can see that the term proportional to the $n$th power contains loop with $n+1$ free propagators. Up to the linear term

$$
I[\mathcal{V}]=I^{(1)}+I^{(2)} \mathcal{V}+\ldots
$$

in terms of the tadpole and of the two propagators loop

$$
\begin{aligned}
I^{(1)} & =G^{(0)}(x, x), \\
I^{(2)}(x-y) & =+2 G_{R}^{(0)}(x, y) G^{(0)}(x, y) .
\end{aligned}
$$

(3) One can easily realize that $I^{(1)}$ diverges as $\Lambda^{2}$ and $\log \Lambda, I^{(2)}$ diverges as $\log \Lambda$ and the loops with more than two propagators are convergent. It is therefore useful to introduce a new functional $J$ containing only the convergent part of $I$ according to

$$
J[\mathcal{V}]=I[\mathcal{V}]-I^{(1)}-I^{(2)} \mathcal{V} .
$$

The previous observations lead us to rewrite the selfconsistent Eq. (3.9) in a particular "quasirenormalized" form, more suitable to analyze the diagrammatic resummation of the effective action and determine its divergent graphs and subgraphs structure. The quasirenormalized form is obtained by expanding the self-consistent Eq. (3.9) around the point $\xi(x)=v^{2}(\mathcal{V}=0)$, explicitly solving the linear terms and writing a self-consistent equation for the higher $\Delta \xi$ powers dependence of $\mathcal{V}$. Doing so one obtains

$$
\mathcal{V}=\frac{1}{2} \theta[\Delta \xi+J[\mathcal{V}]], \quad \theta=\lambda_{0}\left[\mathbf{1}-\frac{1}{2} \lambda_{0} I^{(2)}\right]^{-1} .
$$

We can now use Eqs. (3.11) and (3.5) to calculate the vertex functions relevant for the background equation of motion i.e. those with one $\phi_{\Delta}$ leg at the point $x_{1}$ and $n-1 \phi_{c}$ legs at the points $x_{i}(i>1)$. These are built from the functional derivatives of $\mathcal{F}^{\prime}$ w.r.t. $\xi$ at the point $\xi=v^{2}$. To ease notation we rename such variations by

$$
\Omega^{(k)}\left(x_{1}, \ldots, x_{k}\right)=\left.2 \frac{\delta \mathcal{F}^{\prime}[\xi]\left(x_{1}\right)}{\delta \xi\left(x_{2}\right) \ldots \delta \xi\left(x_{k}\right)}\right|_{\xi=v^{2}} .
$$

We can see that $\Omega^{(1)}=0$. So that $\Gamma^{(1)}=0$, which is just the statement that $v$ is the vacuum solution of the background equation. Moreover we have

$$
\Omega^{(2)}=\frac{1}{2} \theta-\frac{1}{3} \lambda_{0} \mathbf{1}=\frac{1}{6} \lambda_{0} \mathbf{1}-\frac{1}{4} \lambda_{0}^{2} I^{(2)}\left[\mathbf{1}-\frac{1}{2} \lambda_{0} I^{(2)}\right]^{-1} .
$$

Expanding the above expression in powers of $\lambda_{0}$ we see that $\Omega^{(2)}$ is the sum of a classical term $\frac{1}{6} \lambda_{0}$ plus the resummation of all the chains of $I^{(2)}$ loops. The $\Omega^{(k)}$ with $k>2$ are obtained by taking variations of the selfconsistent Eq. (3.11). One can easily see that they are built up with loops having three or more propagators and are therefore finite in the limit $\Lambda \rightarrow \infty$. The loops are attached to each other and to the external legs by the effective vertex $\theta$ [see Eq. (3.11)]. Some graphical examples are
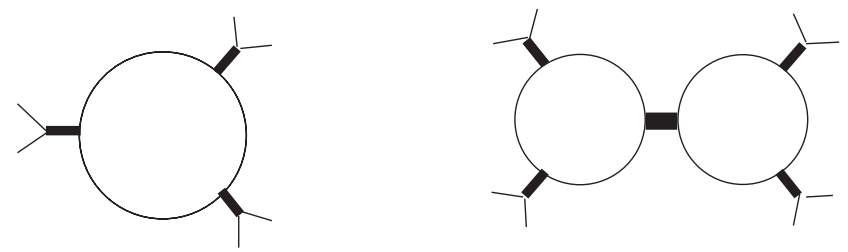

The vertex $\theta$, by its explicit definition in Eq. (3.11), is the sum of the classical term $\lambda_{0}$ plus chains of $I^{(2)}$ integrals.

\section{B. Renormalization}

Let us now apply the standard renormalization procedure to the HF approximation of the effective action. As we will see it fails to render all the vertex functions finite.

The first renormalization condition fixes the physical value $v$ of the vacuum expectation value. Then the gap Eq. (3.2) defines $m_{0}^{2}$ as a function of $\lambda_{0}, v$, and the cutoff $\Lambda$ in such a way to remove all the $\Lambda^{2}$ dependence in the vertex functions. The second renormalization condition fixes instead the value of the quartic coupling at some energy scale $s$ by taking the Fourier transform of the vertex 
$\Gamma^{(4)}$ at the symmetric point $\left(p_{2}, p_{3}, p_{4}\right.$ are the momenta entering the $\phi_{c}$ legs)

$$
-\frac{1}{3} p_{1}=p_{2}=p_{3}=p_{4}=\frac{1}{2} q_{s}, \quad q_{s} \equiv(0, \boldsymbol{q}), \quad|\boldsymbol{q}|=s
$$

and requiring it to be equal to the renormalized coupling constant $\lambda$

$$
\lambda=\tilde{\Gamma}^{(4)}\left(p_{1}, p_{2}, p_{3}\right)=6 \tilde{\Omega}^{(2)}\left(q_{s}\right)=\lambda_{0} \frac{1+\lambda_{0} \tilde{I}^{(2)}\left(q_{s}\right)}{1-\frac{1}{2} \lambda_{0} \tilde{I}^{(2)}\left(q_{s}\right)} .
$$

Notice that in the above formula we have omitted the contributions to the quartic coupling originating from the superficially convergent $\Omega^{(3)}$ and $\Omega^{(4)}$. Compare with the corresponding formula of unbroken symmetry case in Ref. [33] (pay attention to the opposite sign convention for $\left.I^{(2)}\right)$.

As already stated above, this renormalization procedure fails to define a sensible renormalized theory and, in particular, we can individuate the following shortcomings.

The effective quartic coupling $\lambda$ as a function of the bare parameter $\lambda_{0}$ at fixed cutoff $\Lambda$ exhibits an unphysical nonmonotonic behavior that spoils the one-to-one correspondence between bare and renormalized parameters (at fixed cutoff) which holds true only for small coupling. This is to be compared with the standard 1-loop-renormalization-group improved relation which is monotonically increasing with $\lambda_{0}$, at fixed $\Lambda$, as required by physical consistence. Moreover, we see that imposing a finite value to $\lambda$ at the chosen scale $s$ fails to render finite the running coupling (i.e. the generalization of Eq. (3.14) to any value of momentum) at any momentum $p \neq q_{s}$ and even at any $q_{s^{\prime}}$ with $s^{\prime} \neq s$.

For what concerns the higher order terms $\Omega^{(k)}$ with $k>$ 2 we can see that they still depend on $\log \Lambda$ even after renormalization This happens for two specific reasons

(1) The internal (free) propagators mass $m^{2}=\frac{1}{3} \lambda_{0} v^{2}$ introduce an explicit dependence on $\lambda_{0}$. Notice that, unlike the unbroken symmetry case, here the internal propagators differ from the external ones (i.e. the functional inverse of the two-legs vertex function). To the latter contribute also diagrams with chains of $I^{(2)}$ loops as can be seen from its explicit form

$$
\begin{aligned}
\tilde{\Gamma}^{(2)}(p)= & -p^{2}+\frac{1}{3} \lambda(p) v^{2} \\
= & -p^{2}+m^{2}-\frac{1}{2} \lambda_{0}^{2} \tilde{I}^{(2)}(p)[1 \\
& \left.-\frac{1}{2} \lambda_{0} \tilde{I}^{(2)}(p)\right]^{-1} v^{2} .
\end{aligned}
$$

This is finite at momentum $q_{s}$ while, according with what we said above about $\lambda(p)$, logarithmic divergences appear as $p \neq q_{s}$.

(2) In the HF definition of $\Omega^{(k)}$ with $k>2$ there appears the effective vertex $\theta$. By the relation

$$
\tilde{\theta}(p)=\frac{1}{3} \lambda(p)+\frac{2}{3} \lambda_{0}
$$

we can see that an unresolved $\lambda_{0}$ dependence persists even when $p=q_{s}$. For $p \neq q_{s}$ further cutoff dependences appear due to the problems concerning the renormalized running coupling.

We conclude that the HF approximation cannot be renormalized by the standard renormalization procedure: there is an unphysical bare-to-renormalized coupling relation and a plain failure to eliminate divergences in the subgraphs which can be traced to incomplete resummation of Leading Logarithms of the cutoff. In the next subsection we will define a modified HF resummation by explicitly requiring renormalizability and a 1-loop-renormalizationgroup improved relation between $\lambda$ e $\lambda_{0}$.

\section{Improved HF approximation}

As stated in the introduction our recipe for the improvement of the HF resummation consists in two fundamental steps

(1) Fix some HF-likeness properties that we want to maintain throughout the modification and provide a parametrization of the class of approximations having such defining features.

(2) Require explicitly renormalizability and RG invariance in order to fix the form of the arbitrary parameters.

In conclusion, once the result is obtained, we will be able to establish a diagrammatic interpretation of our modified approximation.

The main features of the HF approximation are encoded in the general mean-field form of the background equation of motion, Eq. (2.6) and in the self-consistent definition of $\mathcal{F}^{\prime}$ (through the mean field $\mathcal{V}$ ), Eqs. (3.11). They provide a general recipe for building up all vertex functions using $\Omega^{(2)}$, the effective vertex $\theta$, and the free propagators. Moreover, the self-consistent definition of $\mathcal{F}^{\prime}$ corresponds to the following general form of the equation of motion for the mode functions

$$
\begin{aligned}
& \left\{\square+m^{2}+\mathcal{V}(x)\right\} u_{k}(x)=0, \\
& \mathcal{V}=\frac{1}{2} \theta\left\{\Delta \xi+I-\hat{I}^{(1)}-I^{(2)} \mathcal{V}\right\}
\end{aligned}
$$

with the usual definition of the integral $I$ in terms of the mode functions.

We now regard $\Omega^{(2)}, \theta$, and the effective mass $m^{2}$ of the free propagators as tunable parameters defining a class of approximations that share the same general diagrammatic structure. We abandon the HF definitions for these parameters and look for new ones that ensure proper renormalizability and RG invariance.

We can actually further specify the form of the tunable parameters. By looking at the HF definitions of $\Omega^{(2)}$ and $\theta$ we see that they must have the general leading-log structure 


$$
\Omega^{(2)}=\lambda_{0} F_{1}\left(\lambda_{0} I^{(2)}\right), \quad \theta=\lambda_{0} F_{2}\left(\lambda_{0} I^{(2)}\right)
$$

in terms of two functions, $F_{1}$ and $F_{2}$, of a single variable (the evaluation of these functions on the operator $I^{(2)}$ is obvious if we consider the Fourier transform). We assume that the same holds true for $m^{2}$. This can be understood looking at the explicit form of the two-legs function in Eq. (3.15). We consider $m^{2}$ as a function of $I^{(2)}$ evaluated at zero momentum since we do not want to introduce any new mass scale dependence, that is

$$
m^{2}=\lambda_{0} F_{3}\left(\lambda_{0} \tilde{I}^{(2)}(0)\right) v^{2} .
$$

Notice that changing $\mathrm{m}^{2}$ corresponds to changing the equilibrium solution according to Eq. (3.1). To conclude we observe that the HF approximation resums correctly the 1-loop perturbative order. To maintain this feature we should require the following matching conditions on the F's

$$
\begin{gathered}
F_{1}(x)=\frac{1}{6}+\frac{1}{4} x+O\left(x^{2}\right), \quad F_{2}(x)=1+O(x), \\
F_{3}(x)=\frac{1}{3}+O(x) .
\end{gathered}
$$

Now we have to define our modified HF approximation by fixing new explicit definitions for the functions $F$ 's. We obtain renormalizability with the correct 1-loop beta function, by assuming the following logarithmic dependence for the bare coupling $\lambda_{0}$

$$
\frac{\partial \lambda_{0}}{\partial \log \Lambda}=\frac{3}{16 \pi^{2}} \lambda_{0}^{2}+O\left(\Lambda^{-1}\right)
$$

and requiring that the parameters $\Omega^{(2)}, \theta$, and $m^{2}$ do not depend on $\log \Lambda$. Using

$$
\frac{\partial I^{(2)}}{\partial \log \Lambda}=-\frac{1}{8 \pi^{2}} \mathbf{1}+O\left(\Lambda^{-1}\right)
$$

we have a unique solution that fulfills the matching constraints in Eqs. (3.18),

$$
\begin{aligned}
\theta & =6 \Omega^{(2)}=\lambda_{0}\left[1-\frac{3}{2} \lambda_{0} I^{(2)}\right]^{-1}, \\
m^{2} & =\frac{1}{3} \lambda_{0}\left[1-\frac{3}{2} \lambda_{0} \tilde{I}^{(2)}(0)\right]^{-1} v^{2} .
\end{aligned}
$$

These are the explicit forms of the parameters in our improved HF approximation.

Now applying the renormalization condition we obtain the following bare-to-renormalized relation

$$
\lambda=\frac{\lambda_{0}}{1-\frac{3}{2} \lambda_{0} \tilde{I}^{(2)}\left(q_{s}\right)} .
$$

We can see that our procedure has reproduced the correct 1-loop-renormalization-group improved behavior while at the same time fixing the finite parts. The manifestly finite form for the parameters reads

$$
\begin{aligned}
\theta & =6 \Omega^{(2)}=\lambda\left[1-\frac{3}{2} \lambda J^{(2)}\right]^{-1}, \\
m^{2} & =\frac{1}{3} \lambda\left[1-\frac{3}{2} \lambda \tilde{J}^{(2)}(0)\right]^{-1} v^{2}
\end{aligned}
$$

in terms of $\lambda$ and of the subtracted integral $J^{(2)}$

$$
J^{(2)}=I^{(2)}-\tilde{I}^{(2)}\left(q_{s}\right) \mathbf{1} .
$$

Now, for what concerns RG invariance, one verifies from Eq. (3.20) that the parametrization of $\lambda_{0}$ does not depend on the renormalization scale. In fact, renormalizing at scale $s$ with constant $\lambda=\lambda\left(q_{s}\right)$ or at scale $s^{\prime}$ with constant $\lambda^{\prime}=$ $\lambda\left(q_{s^{\prime}}\right)$ indeed defines the same $\lambda_{0}$. Therefore Eqs. (3.19) can be regarded as manifestly RG-invariant definitions of the parameters.

Finally let us observe that, since $I^{(2)}$ depends on $m^{2}$, we have here an implicit definition of the physical mass by the finite self-consistent relation in Eq. (3.21), rather than an explicit definition, such as the tree-level $m^{2}=\frac{1}{3} \lambda v^{2}$, which can be recovered only if $s=0$.

We give now a brief diagrammatic interpretation of the results just derived. First of all, with the new form of $m^{2}$ in Eq. (3.19), the free propagators are no longer defined as the resummation of tadpole corrections. In fact they are included contributions from graphs as those contributing to HF definition of the external propagator together with all leading-log contributions included in such a way that the $\log \Lambda$ dependence of $m^{2}$ (at fixed $\lambda_{0}$ ) corresponds to the correct 1-loop-renormalization-group improved series. Notice that the contributions of these diagrams are included in a "local" fashion (i.e. with no momentum passing through the loops) in order to maintain the mean-field feature of a momentum-independent self-energy.

Now, for what concerns the new form of $\Omega^{(2)}$ in Eq. (3.19), we can see that it corresponds to the inclusion, in addition to the chain diagrams of the pure HF approximation, of all the leading-log contributions from diagrams to all orders. As before their $\log \Lambda$ dependence is taken while their finite part is fixed by our procedure to be the same of $I^{(2)}$.

In conclusion, the modification of the effective vertex $\theta$ in Eq. (3.19) corresponds to include, in the diagrammatic resummation that defines the $\Omega^{(k)}(k>2)$, leadinglogarithmic contributions from graphs of the form
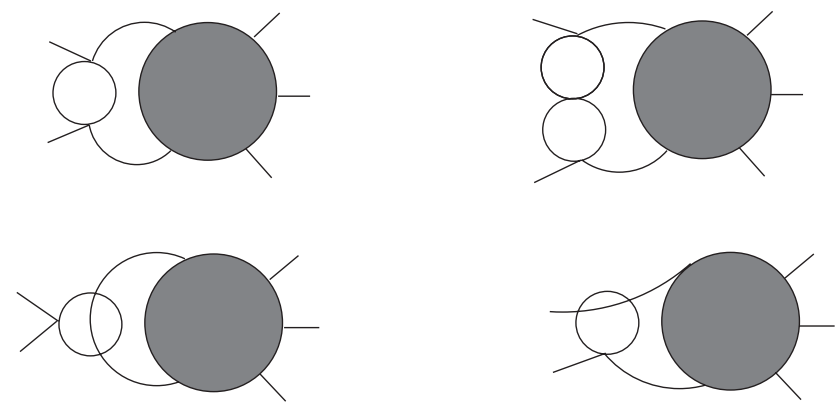

The finite parts of these diagrams are chosen in such a way to maintain the main Hartree-Fock-like features of the effective vertex, namely, the single channel structure and its form as a function of $I^{(2)}$. 
Let us make some observations on the obtained result. The equations of motion of this modified Hartree-Fock approximation read

$$
\begin{aligned}
& \left\{\square+\mathcal{V}(x)-\frac{1}{3} \theta \Delta \xi(x)\right\} \phi(x)=0, \\
& \left\{\square+\frac{1}{3} \tilde{\theta}(0) v^{2}+\mathcal{V}(x)\right\} u_{k}(x)=0, \\
& \mathcal{V}=\frac{1}{2} \theta\left\{\Delta \xi+I-\hat{I}^{(1)}-I^{(2)} \mathcal{V}\right\} .
\end{aligned}
$$

As we can see, in order to obtain renormalizability and RG invariance, we had to introduce space-time nonlocality in the equations of motion. Causality in this nonlocal evolution is guaranteed by the analyticity in the upper half-plane of $\tilde{I}^{(2)}(p)$. Actually in the third equation in Eqs. (3.22) the definition of $\mathcal{V}$ is implicit and it should be solved for $\mathcal{V}$ in order to have a manifestly causal form. This can be done paying the price of losing manifest finiteness

$$
\mathcal{V}=\frac{1}{2} \lambda\left[\mathbf{1}-\lambda\left(I^{(2)}-\frac{3}{2} \tilde{I}^{(2)}\left(q_{s}\right) \mathbf{1}\right)\right]^{-1}\left\{\Delta \xi+I-\hat{I}^{(1)}\right\} .
$$

To conclude we may ask whether and how we can choose different initial conditions for the mode functions without spoiling the properties of renormalizability and RG invariance. We already considered this problem in [33] treating the unbroken symmetry case and the conclusions made there still hold in this case. That is, that all divergent terms are cancelled by renormalization provided one removes the initial time divergences by a Bogoliubov transformation on the initial state (see [38]). Such transformation redefines the initial kernel in such a way that it has the correct shortdistance behavior.

\section{THE CASE $N>1$}

We are now ready to consider the more general case of a scalar field theory with spontaneously broken $O(N)$ symmetry. In doing this we proceed following closely Sec. III.

Before we begin we fix some notational conventions that will help us to handle $O(N)$ index structures while keeping formulas simple and similar to those in Sec. III. In particular we will use the standard matrix notation for objects with one and two indices

$$
\begin{aligned}
& {[M V]_{i}=M_{i j} V_{j}, \quad\left[M^{(1)} M^{(2)}\right]_{i j}=M_{i k}^{(1)} M_{k j}^{(2)},} \\
& \mathbf{1}_{i j}=\delta_{i j}, \quad\left[V V^{T}\right]_{i j}=V_{i} V_{j}, \quad V^{2}=V_{i} V_{i} .
\end{aligned}
$$

When objects with four indices are concerned, we will use the following conventions

$$
\begin{aligned}
& {[T M]_{i j}=T_{i j k m} M_{k m}, \quad\left[T^{(1)} T^{(2)}\right]_{i j k m}=T_{i j r s}^{(1)} T_{r s k m}^{(2)},} \\
& \Pi_{i j k m}=\frac{1}{2}\left(\delta_{i k} \delta_{j m}+\delta_{i m} \delta_{j k}\right), \quad T^{-1} T=T T^{-1}=\Pi,
\end{aligned}
$$

where the last relation is regarded as restricted to tensors with the symmetry $T_{i j k m}=T_{i j m k}=T_{j i k m}$. Moreover we will use also the following definitions

$$
\hat{\mathbf{1}}(x, y)=\mathbf{1} \delta^{(4)}(x-y), \quad \hat{\Pi}(x, y)=\Pi \delta^{(4)}(x-y) .
$$

\section{A. Analysis of the HF approximation}

The Hartree-Fock equations of motion for a scalar $O(N)$ theory are shown in Eqs. (2.4). As a first example of the use of the notational conventions in Eq. (4.1) and Eq. (4.2) we rewrite them in a compact indiceless form

$$
\begin{array}{r}
\left\{\left[\square+m_{0}^{2}+\frac{1}{6} \lambda_{0} \operatorname{tr} \xi\right] \mathbf{1}+\frac{1}{2} \lambda_{0} \tau \int_{p^{2}<\Lambda} \frac{d^{3} p}{(2 \pi)^{3}} u_{p} u_{p}(x)^{\dagger}\right\} \phi=0, \\
\left\{\left[+m_{0}^{2}\right] \mathbf{1}+\frac{1}{2} \lambda_{0} \tau\left[\xi+\int_{p^{2}<\Lambda} \frac{d^{3} p}{(2 \pi)^{3}} u_{p} u_{p}^{\dagger}\right]\right\} u_{\boldsymbol{k}}=0,
\end{array}
$$

where we recall that $\xi(x)=\phi(x) \phi^{T}(x)$ and the definition of $\tau$ in Eq. (2.5).

Exactly as in Sec. III A we select the values of bare parameters corresponding to the broken symmetry phase by requiring the existence of a vacuum solution with nonzero constant and uniform background field, $\phi(x)=v$. Notice that here $v$ is a $O(N)$. The direction $\hat{v}=v / \sqrt{v^{2}}$ of the vacuum background field provides the definition of longitudinal and transverse projectors

$$
P_{L}=\hat{v} \hat{v}^{T}, \quad P_{T}=\mathbf{1}-P_{L} .
$$

The vacuum mode functions now are

$$
\begin{aligned}
u_{\boldsymbol{k}}^{(\mathrm{vac})}(x)= & \left(2 \omega_{\boldsymbol{k}}\right)^{-1 / 2} \exp \left[i(\boldsymbol{k} \cdot \boldsymbol{x}) \mathbf{1}-i \omega_{\boldsymbol{k}} t\right] \\
= & {\left[\left(2 \omega_{\boldsymbol{k}, L}\right)^{-1 / 2} e^{-i \omega_{k, L} t} P_{L}\right.} \\
& \left.+\left(2 \omega_{\boldsymbol{k}, T}\right)^{-1 / 2} e^{-i \omega_{k, T} t} P_{T}\right] e^{i \boldsymbol{k} \cdot \boldsymbol{x}},
\end{aligned}
$$

where in the first line the power and the exponentiation are operations on matrices and $\omega, \omega_{L}$, and $\omega_{T}$ are defined as

$$
\begin{aligned}
\omega_{\boldsymbol{k}}^{2} & =\omega_{\boldsymbol{k}, L}^{2} P_{L}+\omega_{\boldsymbol{k}, T}^{2} P_{T}=\boldsymbol{k}^{2} \mathbf{1}+M^{2}, \\
M^{2} & =m_{L}^{2} P_{L}+m_{T}^{2} P_{T} .
\end{aligned}
$$

The longitudinal mass has the same value as in the $N=1$ case, namely $m_{L}^{2}=\frac{1}{3} \lambda_{0} v^{2}$, while the transverse squared mass $m_{T}^{2}$, as a function of $\lambda_{0}, v^{2}$, and $\Lambda$, is obtained by solving the self-consistent equation

$$
0=m_{T}^{2}+\frac{1}{3} \lambda_{0} \int_{p^{2}<\Lambda} \frac{d^{3} p}{(2 \pi)^{3}}\left\{\frac{1}{2 \omega_{k, L}}-\frac{1}{2 \omega_{k, T}}\right\} .
$$

The vacuum expectation value $v$ satisfies a gap equation which generalizes the one in Eq. (3.2)

$$
\begin{aligned}
0= & m_{0}^{2}+\frac{1}{6} \lambda_{0} v^{2}+\frac{1}{2} \lambda_{0} \int_{p^{2}<\Lambda} \frac{d^{3} p}{(2 \pi)^{3}} \frac{1}{2 \omega_{k, L}} \\
& +\frac{1}{6}(N-1) \lambda_{0} \int_{p^{2}<\Lambda} \frac{d^{3} p}{(2 \pi)^{3}} \frac{1}{2 \omega_{k, T}} .
\end{aligned}
$$


Notice that the gap equation involves only $v^{2}$. In fact varying the direction of $\hat{v}$ we obtain different static solutions that correspond to the equivalent distinct vacua of the theory in the broken symmetry phase.

The mean field $\mathcal{V}$ [see Eq. (3.3)] is now an object with two $O(N)$ indices, defined by

$$
\begin{aligned}
\mathcal{V}(x)= & m_{0}^{2} \mathbf{1}-M^{2}+\frac{1}{2} \lambda_{0} \tau[\xi(x) \\
& \left.+\int_{p^{2}<\Lambda} \frac{d^{3} p}{(2 \pi)^{3}}\left|u_{p}(x)\right|^{2}\right] .
\end{aligned}
$$

Notice that again $\mathcal{V}=0$ on the vacuum solution. In terms of $\mathcal{V}$ Eqs. (4.3) read

$$
\begin{array}{r}
\left\{\square \mathbf{1}+M^{2}-\frac{1}{3} \lambda_{0} \xi+\mathcal{V}\right\} \phi(x)=0, \\
\left\{\square \mathbf{1}+M^{2}+\mathcal{V}\right\} u_{\boldsymbol{k}}(x)=0 .
\end{array}
$$

Recalling the general mean-field form of the background field equation $\left(\mathcal{F}^{\prime}\right.$ here is a $O(N)$ matrix), Eq. (2.6), we have

$$
\mathcal{F}^{\prime}[\xi]=\frac{1}{2} \mathcal{V}+\frac{1}{2} M^{2}-\frac{1}{6} \lambda_{0} \xi
$$

which should be compared with Eq. (3.5) in Sec. III A.

In order to derive a self-consistent equation for $\mathcal{V}$ like the one in Eq. (3.9) we introduce the free mode functions as solutions of the free equation with mass matrix $M$. We also define the free retarded propagator with a straightforward extension of Eq. (3.6) to the case of a mass matrix $M$. Equation (3.7) gives the cutoffed correlation as a functional of $\mathcal{V}$ (now $O(N)$ indices multiplication is understood). In conclusion we have the self-consistent equation for $\mathcal{V}$

$$
\begin{aligned}
\mathcal{V} & =m_{0}^{2} \mathbf{1}-M^{2}+\frac{1}{2} \lambda_{0} \tau(\xi+I[\mathcal{V}]), \\
I[\mathcal{V}](x) & \equiv G[\mathcal{V}](x, x) .
\end{aligned}
$$

This should be compared with Eq. (3.9)

We keep on following closely the Sec. III A by fixing vacuum initial conditions for the mode functions. By this choice follows that $u^{(0)}=u^{(\mathrm{vac})}$ for all times and the free correlation is translationally invariant.

Moreover we can repeat, with little changes, the observations made in Sec. III A about the structure of the integral $I[\mathcal{V}]$. We can still interpret the free Green functions as effective internal propagators obtained by resumming all tadpole corrections to the bare ones. We can introduce the tadpole integral

$$
I^{(1)}=G^{(0)}(x, x)=I_{L}^{(1)} P_{L}+I_{T}^{(1)} P_{T}
$$

and the two propagators loop integral

$$
\begin{aligned}
I_{i j k m}^{(2)}(x-y)= & G_{R, i k}^{(0)}(x-y) G_{m j}^{(0)}(x, y) \\
& +G_{R, m j}^{(0)}(x-y) G_{i k}^{(0)}(x, y) \\
= & I_{L L}^{(2)}(x-y) P_{L, i k} P_{L, j m}+I_{T T}^{(2)}(x-y) P_{T, i k} P_{T, j m} \\
& +\frac{1}{2} I_{T L}^{(2)}(x-y)\left(P_{L, i k} P_{T, j m}+P_{T, i k} P_{L, j m}\right) .
\end{aligned}
$$

In terms of these Eq. (3.10) defines the functional $J$ which contains only the cutoff convergent part of $I$ [here $O(N)$ indices contraction according to the rules in Eq. (4.2) is understood]. Notice also that, by using the two propagator loop integral of Eq. (4.7), we can cast the self-consistent definition of $m_{T}^{2}$ in Eq. (4.4) into a more compact form

$$
m_{T}^{2}=-\frac{1}{3} \lambda_{0} \tilde{I}_{T L}^{(2)}(0) m_{T}^{2}\left[1-\frac{1}{3} \lambda_{0} \tilde{I}_{T L}^{(2)}(0)\right]^{-1} .
$$

The quasirenormalized form is obtained by manipulations identical to those in Sec. III A

$$
\begin{aligned}
\mathcal{F}^{\prime} & =\frac{1}{2} \Omega^{(1)}+\left[\frac{1}{2} \Omega^{(2)}-\frac{1}{4} \theta\right] \Delta \xi+\frac{1}{2} \mathcal{V}, \\
\mathcal{V} & =\frac{1}{2} \theta \Delta \xi+\frac{1}{2} \theta J[\mathcal{V}] .
\end{aligned}
$$

The matrix $\Omega^{(1)}$ and the four-indices objects $\Omega^{(2)}$ and $\theta$ are defined as

$$
\begin{gathered}
\Omega^{(1)}=m_{T}^{2} P_{T}, \quad \theta=\lambda_{0} \tau\left[\hat{\Pi}-\frac{1}{2} \lambda_{0} \tau I^{(2)}\right]^{-1}, \\
\Omega^{(2)}=\frac{1}{2} \theta-\frac{1}{3} \lambda_{0} \hat{\Pi} .
\end{gathered}
$$

The vertex functions contributing to the equation of motion can be expressed in terms of functional derivatives of $\mathcal{F}^{\prime}$

$$
\Omega_{i_{1} j_{1} \ldots i_{k} j_{k}}^{(k)}\left(x_{1}, \ldots, x_{k}\right)=\left.2 \frac{\delta \mathcal{F}^{\prime}[\xi]\left(x_{1}\right)}{\delta \xi_{i_{1}, j_{1}}\left(x_{2}\right) \ldots \delta \xi_{i_{k} j_{k}}\left(x_{k}\right)}\right|_{\xi=v^{2}}
$$

as in Sec. III A. Notice that $\Omega^{(1)}$ is completely transverse [see Eq. (4.9)] which implies that $\Gamma^{(1)}=\Omega^{(1)} v=0$, namely, the statement that the vector $v$ is the vacuum static solution.

Of course all the diagrammatic interpretations made in Sec. III A still hold true, now with diagrams carrying $O(N)$ indices.

Before going further, we should introduce some notations that will be useful later on. Four-indices tensors, functions of $v$, and of other scalar quantities, with the following symmetry properties (e.g. $\theta$ and $\Omega^{(2)}$ ) admit a general decomposition

$$
\begin{aligned}
& T_{i j k m}(v)=T_{j i k m}(v)=T_{i j m k}(v)=T_{k m i j}(v), \\
& T_{i j k m}(v)=T_{\alpha}\left(v^{2}\right) t_{i j k m}^{\alpha}(\hat{v})
\end{aligned}
$$

in terms of the five elementary tensors

$$
\begin{aligned}
t_{i j k m}^{1}= & \hat{v}_{i} \hat{v}_{j} \hat{v}_{k} \hat{v}_{m}, \quad t_{i j k m}^{2}=\frac{1}{2}\left(P_{T, i k} P_{T, j m}+P_{T, i m} P_{T, j k}\right), \\
t_{i j k m}^{3}= & P_{T, i j} P_{T, k m}, \quad t_{i j k m}^{5}=\left(P_{L, i j} P_{T, k m}+P_{T, i j} P_{L, k m}\right), \\
t_{i j k m}^{4}= & \frac{1}{2}\left(P_{L, i k} P_{T, j m}+P_{L, i m} P_{T, j k}+P_{T, i k} P_{L, j m}\right. \\
& \left.+P_{T, i m} P_{L, j k}\right) .
\end{aligned}
$$

Notice that the coefficients of the decomposition are functions of $v^{2}$ alone. As an example, by Eq. (4.9) the coefficients $\theta_{\alpha}$ of the decomposition of $\theta$ are 


$$
\begin{aligned}
\theta_{1}= & \lambda_{0}\left[\mathbf{1}-\frac{1}{9}(N+2) \lambda_{0} I_{T T}^{(2)}\right]\left\{\mathbf{1}-\frac{1}{6}(N+1) \lambda_{0} I_{T T}^{(2)}\right. \\
& \left.-\frac{1}{2} \lambda_{0} I_{L L}^{(2)}\left[\mathbf{1}-\frac{1}{9}(N+2) \lambda_{0} I_{T T}^{(2)}\right]\right\}^{-1}, \\
\theta_{2}= & \frac{2}{3} \lambda_{0}\left[\mathbf{1}-\frac{1}{3} \lambda_{0} I_{T T}^{(2)}\right]^{-1} \\
\theta_{4}= & \frac{2}{3} \lambda_{0}\left[\mathbf{1}-\frac{1}{3} \lambda_{0} I_{T L}^{(2)}\right]^{-1} \\
\theta_{5}= & \frac{1}{3} \theta_{1}\left[\mathbf{1}-\frac{1}{9}(N+2) \lambda_{0} I_{T T}^{(2)}\right]^{-1}, \\
\theta_{3}= & \theta_{5}\left[\mathbf{1}-\frac{1}{3} \lambda_{0} I_{L L}^{(2)}\right]\left[\hat{1}-\frac{1}{3} \lambda_{0} I_{T T}^{(2)}\right]^{-1} .
\end{aligned}
$$

Notice that using Eqs. (4.11) the compact form of the selfconsistent definition of $m_{T}^{2}$ in Eq. (4.8) can be rewritten as

$$
m_{T}^{2}=\frac{1}{3} \lambda_{0} v^{2}-\frac{1}{2} \tilde{\theta}_{4}(0) v^{2} .
$$

The coefficient of $\Omega$ can be determined from those of $\theta$ by Eq. (4.9) and the decomposition of the $\Pi$ tensor.

\section{B. Renormalization}

Now we apply the standard renormalization procedure as we did in Sec. III B for the $N=1$ case. We will see that the same problems are present also in this case and some others will arise due to the presence of the spontaneously broken continuous $O(N)$ symmetry.

By fixing the equilibrium value of the background field we provide the first renormalization condition that defines the bare mass $m_{0}^{2}$ as a function of $\lambda_{0}, v$, and $\Lambda$. This removes all $\Lambda^{2}$ dependence from the vertex functions.

The second renormalization condition is conventionally obtained by evaluating at the symmetric point [see Eq. (3.13)] the "all-longitudinal" component of the fourlegs vertex function $\Gamma^{(4)}$ and requiring it to be equal to the renormalized coupling $\lambda$

$$
\begin{aligned}
\lambda= & 6 \tilde{\Omega}_{1}^{(2)}\left(q_{s}\right) \\
= & \frac{3 \lambda_{0}\left[1-\frac{N+2}{9} \lambda_{0} \tilde{I}_{T T}^{(2)}\left(q_{s}\right)\right]}{1-\frac{N+1}{6} \lambda_{0} \tilde{I}_{T T}^{(2)}\left(q_{s}\right)-\frac{1}{2} \lambda_{0} \tilde{I}_{L L}^{(2)}\left(q_{s}\right)\left[1-\frac{N+2}{9} \lambda_{0} \tilde{I}_{T T}^{(2)}\left(q_{s}\right)\right]} \\
& -2 \lambda_{0},
\end{aligned}
$$

where, as in Sec. III B we have dropped the contributions from $\Omega^{(3)}$ and $\Omega^{(4)}$. This has to be compared with the corresponding relation of the $N=1$ case.

First of all Eq. (4.13) shows the same pathological dependence of $\lambda$ on $\lambda_{0}$, at fixed $\Lambda$ and $v$, that we found in Sec. III B. As already discussed this prevents a consistent map between bare parameters and renormalized ones unless we restrict to a small coupling regime. For what concerns the cutoff dependence of $\Omega^{(2)}$ we can see that not only the $\log \Lambda$ dependence is not removed by the renormalization condition from $\tilde{\Omega}^{(2)}(p)$ for $p \neq q_{s}$, but also, for components $\Omega_{\alpha}$ with $\alpha \neq 1$ logarithmic divergences already appear at $p=q_{s}$. Regarding the cutoff dependence of the $\Omega^{(k)}$ with $k>2$ as in Sec. III B renormalization does not remove $\log \Lambda$ dependence in the masses of the free propagators and in the effective vertex $\theta$.
Besides these renormalization problems analogous to the $N=1$ case, another very important aspect, which is peculiar of the $N>1$ case, must be pointed out. Equations (4.3) are manifestly $O(N)$ symmetric and so it should be for the effective action. Therefore the standard Ward identity, stating the masslessness of the external transverse propagator (the inverse of $\tilde{\Gamma}_{T}^{(2)}(p)$ ), is indeed satisfied by the HF approximation

$$
\begin{aligned}
\Gamma^{(2)} & =\Gamma_{L}^{(2)} P_{L}+\Gamma_{T}^{(2)} P_{T}, \\
\tilde{\Gamma}_{L}^{(2)}(p) & =-p^{2}+2 \tilde{\Omega}_{1}^{(2)}(p) v^{2}, \\
\tilde{\Gamma}_{T}^{(2)}(p) & =-p^{2}+\Omega_{T}^{(1)}+\tilde{\Omega}_{4}^{(2)}(p) v^{2} \\
& =-p^{2}+m_{T}^{2}+\left[\frac{1}{2} \tilde{\theta}_{4}(p)-\frac{1}{3} \lambda_{0}\right] v^{2}
\end{aligned}
$$

thanks to the self-consistent definition of the transverse mass, Eq. (4.12). But the transverse mass itself, which enters the internal transverse propagators $G_{R, T}^{(0)}$ and $G_{T}^{(0)}$ does not vanish at all (it actually diverges as $\log \Lambda$ ), preventing a consistent interpretation of the transverse modes as Goldstone bosons. This is a well-known problem of the HF approximation (see for instance Ref. [35]).

\section{A class of improved HF approximations}

We shall now try and improve the HF approximation to recover the correct properties of renormalizability and RG invariance. We shall also require that this improved resummation is gapless; that is, we shall impose that the internal transverse propagators are massless. To this end we follow closely the procedure of Sec. IIIC, while stressing some important new features which appear due to the continuous $O(N)$ symmetry and the presence of two kinds of fields (transverse and longitudinal) with different masses. Let us anticipate that in this case our procedure does not single out a unique solution but rather an extended class of resummations that share all the required properties.

As in Sec. IIIC the first step consists in fixing some fundamental properties of the HF approximation that we want to preserve. First of all the general structure of the diagrams resummation which is encoded in the general mean-field form of the background equations of motion, Eq. (2.6), and in the self-consistent definition of $\mathcal{F}^{\prime}$, Eqs. (4.6). The latter implies the following mean-fieldtype equations of motion for the mode functions

$$
\begin{aligned}
{\left[\square \mathbf{1}+M^{2}+\mathcal{V}\right] u_{k} } & =0, \\
\mathcal{V} & =\frac{1}{2} \theta\left\{\Delta \xi+I-I^{(1)}-I^{(2)} \mathcal{V}\right\} .
\end{aligned}
$$

As in Sec. III C we now regard $\Omega^{(1)}, \Omega^{(1)}, \theta$, and the free propagators masses $m_{L}^{2}$ and $m_{T}^{2}$ (in matrix form $M^{2}$ ) as tunable parameters that we are going to change with regard to (w.r.t.) to their HF definitions.

The first important difference with the $N=1$ case is that now $\Omega^{(2)}, \theta$, and $M$ are not independent, but must fulfill certain $O(N)$ symmetry constraints. In fact the equations of 
motion covariant under contemporaneous rotations of the field $\phi$, of the mode functions $u_{p}$, and of the vacuum expectation value $v$ which enters in the definitions the propagators and of $\Omega^{(2)}$ and $\theta$. We have to explicitly require symmetry under rotations of $\phi$ and $u_{p}$ alone or, equivalently, invariance under rotations of $v$. Explicitly we have to impose

$$
\delta\left[M^{2}+\mathcal{V}\right]=0, \quad \delta \mathcal{F}^{\prime}=0,
$$

where $\delta$ is the operator generating the infinitesimal variation under an $O(N)$ rotation of $v$. Equations (4.16) require invariance in the mode functions Eqs. (4.15) and of the background field equation, Eq. (2.6), respectively. Solving explicitly for $\mathcal{V}$ the second equation in Eqs. (4.15) we can rewrite the first condition in Eqs. (4.16) as

$$
0=\delta M^{2}+\delta\left\{\left[\mathbf{1}+\frac{1}{2} \theta I^{(2)}\right]^{-1} \theta\left[\Delta \xi+I-I^{(1)}\right]\right\} .
$$

Notice that $\xi$ and $u_{k}$ are independent and by hypothesis invariant variables (i.e. they must depend only on $v^{2}$ ). Then Eq. (4.17) implies

$$
\begin{aligned}
\delta\left\{\left[\mathbf{1}+\frac{1}{2} \theta I^{(2)}\right]^{-1} \theta\right\}= & 0, \\
0= & \delta M^{2}+\left[\mathbf{1}+\frac{1}{2} \theta I^{(2)}\right]^{-1} \\
& \left.\times \theta\left[-v^{2} \delta P_{L}-\delta I^{(1)}\right]\right\} .
\end{aligned}
$$

By some easy algebraic manipulations we can rewrite the first equation above as

$$
\delta \theta=\frac{1}{2} \theta \delta I^{(2)} \theta
$$

which has a simple and natural diagrammatic interpretation. Making the tensorial structure explicit, we obtain the following conditions on the coefficients $\theta_{\alpha}$

$$
\begin{aligned}
& \theta_{2}=\theta_{4}\left[\mathbf{1}-\frac{1}{2}\left(I_{T T}^{(2)}-I_{T L}^{(2)}\right)\right]^{-1}, \\
& \theta_{5}=\left[\theta_{1}-\theta_{4}+\frac{1}{2} \theta_{4} \theta_{1}\left(I_{L L}^{(2)}-I_{T L}^{(2)}\right)\right]\left[\mathbf{1}+\frac{1}{2} \theta_{4}\left(I_{T T}^{(2)}-I_{T L}^{(2)}\right)\right]^{-1}, \\
& \theta_{3}=\theta_{5}\left[\mathbf{1}+\frac{1}{2} \theta_{4}\left(I_{L L}^{(2)}-I_{T L}^{(2)}\right)\right]\left[\mathbf{1}+\frac{1}{2} \theta_{4}\left(I_{T T}^{(2)}-I_{T L}^{(2)}\right)\right]^{-1} .
\end{aligned}
$$

Notice that these relations uniquely fix the form of $\theta$ once $\theta_{1}$ and $\theta_{4}$ have been specified. Now, using these relations and the following rules for the explicit variations

$$
\begin{aligned}
& \delta M^{2}=\left(m_{L}^{2}-m_{T}^{2}\right) \delta P_{L}, \\
& \delta I^{(1)}=\left(I_{L}^{(1)}-I_{T}^{(1)}\right) \delta P_{L}=\left(m_{L}^{2}-m_{T}^{2}\right) \tilde{I}_{T L}^{(2)}(0) \delta P_{L}
\end{aligned}
$$

we can rewrite the second equation in Eqs. (4.18) as

$$
m_{L}^{2}-m_{T}^{2}=\frac{1}{2} v^{2} \tilde{\theta}_{4}(0)
$$

The second condition in Eqs. (4.16) can be written as

$$
\delta \Omega^{(2)}=\frac{1}{2} \delta \theta, \quad \delta \Omega^{(1)}-\delta M^{2}-\left(\Omega^{(2)}-\frac{1}{2} \theta\right) v^{2} \delta P_{L} .
$$

Explicit tensorial calculation yields

$$
\begin{aligned}
& \Omega_{2}^{(2)}=\Omega_{4}^{(2)}+\frac{1}{2}\left[\theta_{2}-\theta_{4}\right], \\
& \Omega_{3}^{(2)}=\Omega_{1}^{(2)}-\Omega_{4}^{(2)}+\frac{1}{2}\left[\theta_{3}-\theta_{1}+\theta_{4}\right], \\
& \Omega_{5}^{(2)}=\Omega_{1}^{(2)}-\tilde{\Omega}_{4}^{(2)}+\frac{1}{2}\left[\theta_{5}-\theta_{1}+\theta_{4}\right], \\
& \Omega^{(1)}=-\tilde{\Omega}_{4}^{(2)}(0) v^{2} P_{T} .
\end{aligned}
$$

In conclusion, according to Eqs. (4.19), (4.20), and (4.21), the actual independent parameters are $\theta_{1}, \theta_{4}, m_{L}^{2}, \Omega_{1}^{(2)}$, and $\Omega_{4}^{(2)}$. The integrated versions of Eqs. (4.16) are obtained by writing $M^{2}+\mathcal{V}$ and $\mathcal{F}^{\prime}$ in a manifestly $O(N)$ symmetric form

$$
\begin{aligned}
M^{2}+\mathcal{V} & =\mu^{(1)} \mathbf{1}+\frac{1}{2} \gamma^{(1)}[\xi+I], \\
\mathcal{F}^{\prime} & =\mu^{(2)} \mathbf{1}+\frac{1}{2} \gamma^{(2)} \xi+\frac{1}{2}\left[M^{2}+\mathcal{V}\right], \\
\gamma_{i j k m}^{(\alpha)} & =\gamma_{1}^{(\alpha)} \delta_{i j} \delta_{k m}+\frac{1}{2} \gamma_{2}^{(\alpha)}\left[\delta_{i k} \delta_{j m}+\delta_{i m} \delta_{j k}\right], \\
\alpha & =1,2
\end{aligned}
$$

with $\gamma^{(\alpha)}$ and $\mu^{(\alpha)}$ defined in terms of the free parameters as follows

$$
\begin{aligned}
\gamma_{1}^{(1)} & =\left[\theta_{1}-\theta_{4}-\frac{1}{2} \theta_{4} \theta_{1}\left(I_{L L}^{(2)}-I_{T L}^{(2)}\right)\right]\left[\mathbf{1}+\frac{1}{2} \theta_{4}\left(I_{T L}^{(2)}-I_{T T}^{(2)}\right)\right]\left[\mathbf{1}-\frac{1}{2} \theta_{4} I_{T L}^{(2)}\right]^{-1} \Delta^{-1}, \\
\Delta & =\left[\mathbf{1}+\frac{1}{2} \theta_{4}\left[I_{T L}^{(2)}-N I_{T T}^{(2)}\right]\right]\left[\mathbf{1}+\frac{1}{2} \theta_{1} I_{L L}^{(2)}\right]-\frac{N-1}{2} \theta_{1} I_{T T}^{(2)}\left[\mathbf{1}+\frac{1}{2} \theta_{4} I_{T L}^{(2)}\right]^{-1}, \\
\gamma_{2}^{(1)} & =\theta_{4}\left[\mathbf{1}+\frac{1}{2} I_{T L}^{(2)}\right]^{-1} \\
\gamma_{1}^{(2)} & =\frac{1}{2} \Omega_{3}^{(2)}-\frac{1}{4} \theta_{3} \\
\gamma_{2}^{(2)} & =\frac{1}{2} \Omega_{4}^{(2)}-\frac{1}{4} \theta_{4} \\
\mu^{(1)} & =m_{L}^{2}-\frac{1}{2} \tilde{\gamma}_{2}^{(1)}(0)\left[v^{2}+I_{L}^{(1)}\right]-\frac{1}{2} \tilde{\gamma}_{1}^{(1)}(0)\left[v^{2}+I_{L}^{(1)}+(N-1) I_{T}^{(1)}\right] \\
\mu^{(2)} & =-\frac{1}{2} m_{L}^{2}-\frac{1}{2} \tilde{\Omega}_{1}^{(2)}(0) v^{2}+\frac{1}{4} \tilde{\theta}_{1}(0) v^{2} .
\end{aligned}
$$

One can verify that the standard HF definitions of the tunable parameters indeed satisfy these conditions.

Now, as in Sec. III C, we require that $\theta$ and $\Omega^{(2)}$ have the same general leading-log structure characteristic of their HF definitions namely 


$$
\begin{gathered}
\Omega_{1}^{(2)}=\lambda_{0} F_{1}\left(\lambda_{0} I_{L L}^{(2)}, \lambda_{0} I_{T L}^{(2)}, \lambda_{0} I_{T T}^{(2)} ; N\right), \\
\theta_{1}=\lambda_{0} F_{2}\left(\lambda_{0} I_{L L}^{(2)}, \lambda_{0} I_{T L}^{(2)}, \lambda_{0} I_{T T}^{(2)} ; N\right), \\
\Omega_{4}^{(2)}=\lambda_{0} F_{3}\left(\lambda_{0} I_{L L}^{(2)}, \lambda_{0} I_{T L}^{(2)}, \lambda_{0} I_{T T}^{(2)} ; N\right), \\
\theta_{4}=\lambda_{0} F_{4}\left(\lambda_{0} I_{L L}^{(2)}, \lambda_{0} I_{T L}^{(2)}, \lambda_{0} I_{T T}^{(2)} ; N\right),
\end{gathered}
$$

where the $F_{A}(x, y, z ; N)$ are generic functions of commuting arguments since the operators $I_{L L}^{(2)}, I_{T L}^{(2)}$, and $I_{T T}^{(2)}$ are diagonal in Fourier space. We assume a similar structure also for $m_{L}^{2}$ as we did in Eq. (3.17) for the $N=1$ case

$$
m_{L}^{2}=\lambda_{0} v^{2} F_{5}\left(\lambda_{0} \tilde{I}_{L L}^{(2)}(0), \lambda_{0} \tilde{I}_{T L}^{(2)}(0), \lambda_{0} \tilde{I}_{T T}^{(2)}(0) ; N\right) .
$$

We have thus reduced our parametrization freedom to five functions $F_{A}$ of three variables. We still have to require that these functions fulfill some further properties that hold true in the HF approximation.

Hartree-Fock matches perturbatively at 1-loop order. This requires that $\theta$ and $m_{L}^{2}$ match at tree level and that $\Omega^{(2)}$ matches at 1-loop order (the tree-level term never appears in the vertex functions). Explicitly we have

$$
\begin{aligned}
& F_{1}(x, y, z ; N)=\frac{1}{6}+\frac{1}{4} x+\frac{1}{36}(N-1) z+\ldots, \\
& F_{3}(x, y, z ; N)=c_{0}+\frac{1}{9} y+\ldots, \\
& F_{2}(x, y, z ; N)=1+\ldots, \\
& F_{4}(x, y, z ; N)=\frac{2}{3}+\ldots, \\
& F_{5}(x, y, z ; N)=\frac{1}{3}+\ldots,
\end{aligned}
$$

where $c_{0}$ is a purely numerical arbitrary constant.

In the $N \rightarrow \infty$ limit the HF resummation reproduces correctly the equations of the usual large $N$ approximation. This can be done as follows. First we restrict to the special case of a background field which maintains a fixed direction (i.e. the direction of the vacuum expectation value $v$ ), that is $\phi_{i}(x)=\phi(x) \hat{v}_{i}$. Then we can reduce the equations of motion into a projected form

$$
\begin{aligned}
& {\left[\square+\mathcal{V}_{L}+\left(\Omega_{1}^{(2)}-\frac{1}{2} \theta_{1}\right) \Delta \xi\right] \phi=0,} \\
& {\left[\square+m_{T}^{2}+\mathcal{V}_{T}\right] u_{k T}=0, \quad\left[\square+m_{L}^{2}+\mathcal{V}_{L}\right] u_{k L}=0,} \\
& \mathcal{V}_{L}=\frac{1}{2} \theta_{1}\left[\Delta \xi+J_{L}\right] \quad+\frac{1}{2}(N-1) \theta_{5} J_{T}, \\
& \mathcal{V}_{T}=\frac{1}{2} \theta_{5}\left[\Delta \xi+J_{L}\right] \quad+\left[\frac{1}{2}(N-1) \theta_{3}+\frac{1}{2} \theta_{2}\right] J_{T} .
\end{aligned}
$$

Now let us rescale the coupling, the background field, and the vacuum expectation value as prescribed by the standard large $N$ procedure and then by taking the limit and using the symmetry conditions one can see that the correct large $N$ equations are recovered, provided the limits of the free parameters satisfy the following relations

$$
\begin{aligned}
\frac{1}{2} v^{2} \tilde{\theta}_{4}(0)-m_{L}^{2} & \rightarrow 0, \\
N\left(\theta_{1}-\theta_{4}\right) & \rightarrow \frac{1}{3} \lambda_{0}\left[\mathbf{1}-\frac{1}{6} \lambda_{0} I_{T T}^{(2)}\right]^{-1}, \\
N \Omega_{1}^{(2)} & \rightarrow \frac{1}{6} \lambda_{0}\left[\mathbf{1}-\frac{1}{6} \lambda_{0} \tilde{I}_{T T}^{(2)}\right]^{-1} .
\end{aligned}
$$

Suitable conditions on the functions $F_{A}$ then follow from Eqs. (4.23). Notice that in the integrals $I_{T T}^{(2)}$ in Eqs. (4.25) we have $m_{T}^{2}=0$, as consistent with the limits of the parameters.

To conclude, in the HF approximation $\Omega_{1}^{(2)}$ and the $\theta_{\alpha}$ fulfill positivity conditions. More precisely they are real and positive when evaluated at the purely spatial value of the momentum $q_{s}$ [see Eq. (3.13)].

We now proceed in defining our modified HF approximation by making some further sensible requirements that are not satisfied by the HF approximation. First of all we require our approximation to be gapless. That is to say we require that the transverse mass of the internal propagator is zero. As a consequence by Eq. (4.20) we have

$$
m_{L}^{2}=\frac{1}{2} v^{2} \theta_{4}(0) \longrightarrow F_{5}(x, y, z ; N)=\frac{1}{2} F_{4}(x, y, z ; N) .
$$

Because of this condition the transverse internal propagators are now massless and therefore the Goldstone bosons loop integral $I_{T T}^{(2)}$ is logarithmically IR-divergent. The symmetry relations in Eq. (4.26) and Eq. (4.21) define the longitudinal mass $m_{L}^{2}$ and $\Omega^{(1)}$ in terms of the zero momentum values of $\theta_{4}$ and $\Omega_{4}^{(2)}$, respectively. To avoid IR divergences we then require that $\theta_{4}$ and $\Omega_{4}^{(2)}$ do not depend at all on $I_{T T}^{(2)}$. Thus we can write

$$
\begin{aligned}
& F_{3}(x, y, z ; N)=K_{3}(x, y ; N), \\
& F_{4}(x, y, z ; N)=K_{4}(x, y ; N),
\end{aligned}
$$

where $K_{A}(x, y ; N)(A=3,4)$ are arbitrary functions of two variables only (plus $N$ ). The same argument applies to the parameters $\mu^{(1)}$ and $\mu^{(2)}$ of the manifestly symmetric form in Eq. (4.22): we require that $\frac{1}{2} \theta_{1}-\Omega_{1}^{(2)}$ and $\gamma_{2}^{(2)}$ do not depend on $I_{T T}^{(2)}$, which in turn implies

$$
\begin{aligned}
F_{2}(x, y, z ; N)= & \frac{K_{4}(x, y ; N)}{1+\frac{1}{2} K_{4}(x, y ; N)(y-x)} \\
& -\frac{K_{4}(x, y ; N)}{(N-1) \frac{\left[1+\frac{1}{2} K_{4}(x, y ; N)(y-x)\right]^{2}}{1+\frac{1}{2} K_{4}(x, y ; N)(y-z)}+K_{2}(x, y ; N)}, \\
F_{1}(x, y, z ; N)= & -\frac{1}{2} \\
& \times \frac{K_{4}(x, y ; N)}{(N-1) \frac{\left[1+\frac{1}{2} K_{4}(x, y ; N)(y-x)\right]^{2}}{1+\frac{1}{2} K_{4}(x, y ; N)(y-z)}+K_{2}(x, y ; N)} \\
& +K_{1}(x, y ; N),
\end{aligned}
$$

where, again, $K_{A}(x, y ; N)(A=1,2)$ are arbitrary functions of two variables (and $N$ ). Now, as in Sec. III C, we require the renormalizability with the 1-loop beta function. That is, we assume the following RG equation for the bare coupling $\lambda_{0}$ 


$$
\frac{\partial \lambda_{0}}{\partial \log \Lambda}=\frac{N+8}{24 \pi^{2}} \lambda_{0}^{2}+O\left(\Lambda^{-1}\right)
$$

and ask that the free parameter function $K_{A}$ do not depend on $\log \Lambda$. By the same procedure of Sec. III C and using

$$
\begin{aligned}
\frac{\partial I_{L L}^{(2)}}{\partial \log \Lambda} & =\frac{\partial I_{T T}^{(2)}}{\partial \log \Lambda}+O\left(\Lambda^{-1}\right)=\frac{\partial I_{T L}^{(2)}}{\partial \log \Lambda}+O\left(\Lambda^{-1}\right) \\
& =-\frac{1}{8 \pi^{2}} \mathbf{1}+O\left(\Lambda^{-1}\right),
\end{aligned}
$$

we obtain the general forms

$$
\begin{gathered}
K_{j}(x, y ; N)=\frac{f_{j}(\alpha(x, y) ; N)}{1-\frac{1}{6}(N+8) x}, \quad j=1,3,4, \\
K_{2}(x, y ; N)=f_{2}(\alpha(x, y) ; N),
\end{gathered}
$$

where

$$
\alpha(x, y)=\frac{x-y}{1-\frac{1}{6}(N+8) x} .
$$

Recalling that $x=\lambda_{0} I_{L L}^{(2)}$ and $y=\lambda_{0} I_{T L}^{(2)}$ we see that the Fourier transform of $\alpha$ positive definite for purely spatial value of the momentum. Notice also that $\alpha$ vanishes when $v \rightarrow 0$ since $I_{L L}^{(2)}$ and $I_{T L}^{(2)}$ coincide in this limit.

Notice that now, in contrast to the $N=1$ case studied in Sec. III C [see Eqs. (3.19)], the requirement of renormalizability does not fix completely the form of the free parameters. This occurs because for $N>1$ there exists three distinct finite parts associated with the logarithmic cutoff divergence of $I_{L L}^{(2)}, I_{T L}^{(2)}$, and $I_{T T}^{(2)}$. So now we are left with four arbitrary functions $f_{A}$ of one variable (and $N$ ) to determine. One last requirement is that the $N=1$ case should be recovered for all the parameters that have meaning also in this case. These are $\Omega_{1}^{(2)}, \theta_{1}$, and $\theta_{4}$ (that, evaluated at zero momentum, determines the longitudinal mass according to Eq. (4.26)). When $N=1$ they should have the following form [see Eqs. (3.19)]

$$
\theta_{1}=12 \Omega_{1}^{(2)}=\frac{3}{2} \theta_{4}=\lambda_{0}\left[\mathbf{1}-\frac{3}{2} \lambda_{0} I_{L L}^{(2)}\right]^{-1}
$$

that is, in terms of the functions $f$ 's,

$$
\begin{gathered}
f_{4}(\alpha ; 1)=\frac{2}{3}, \quad f_{2}(\alpha ; 1)=-\frac{2-\frac{2}{3} \alpha}{1-\alpha}, \\
f_{1}(\alpha ; 1)=\frac{\alpha}{9-3 \alpha} .
\end{gathered}
$$

Notice also that all the possible choices of free parameters coincide when $v \rightarrow 0$, since $\alpha$ vanishes in this limit and the perturbative constraints uniquely fix the form of the $f$ 's when $\alpha=0$. Moreover one can verify that the resulting $v=0$ improved HF coincide with the massless limit of the improved approximation in the unbroken symmetry phase defined in Ref. [33].

In conclusion all possible forms of the functions $f$ 's that fulfill the above matching constraints define improved
Hartree-Fock resummations with the required features of gaplessness and renormalizability. One simple choice for the $f$ 's is

$$
\begin{aligned}
& f_{4}(\alpha ; N)=2 / 3, \\
& f_{2}(\alpha ; N)=-(N+1) \frac{1-\frac{1}{3} \alpha}{1-\alpha}+(N-1) \alpha, \\
& f_{1}(\alpha ; N)=\frac{\alpha}{9-2 \alpha}, \\
& f_{3}(\alpha ; N)=\frac{2}{3(N+8)} \frac{1}{1+\frac{N+8}{6} \alpha} .
\end{aligned}
$$

As already remarked, this choice is not unique. For example we can consider a second form

$f_{4}(\alpha ; N)=\frac{2 / 3}{1+\frac{N-1}{6} \alpha}$,

$f_{2}(\alpha ; N)=-(N+1) \frac{1-\frac{1}{3} \alpha}{1-\alpha}+\frac{4(N-1)}{3} \alpha \frac{1+\frac{N+8}{12} \alpha}{1+\frac{N+8}{6} \alpha}$,

$f_{1}(\alpha ; N)=\frac{\alpha}{9-2 \alpha}$,

$f_{3}(\alpha ; N)=\frac{2}{3(N+8)} \frac{1}{1+\frac{N+8}{6} \alpha}$.

As already explained in Sec. III C, our modified resummation adds leading-logarithm contributions of diagrams that are not present in the usual HF approximation; then the two forms just provided of the free parameters, as well as all the other possible ones, correspond to different choices of the associated finite parts.

Given one specific choice for the free parameters, we can proceed in applying the coupling constant renormalization condition in Eq. (4.13). Notice that the consistence of this renormalization requires that $\Omega_{1}^{(2)}$ is monotonically growing with $\lambda_{0}$ for any given purely spatial momentum. We have omitted to include this requirement in the previous general discussion since it would lead in general to rather complicated constraints. It holds true for the two simple examples given in Eqs. (4.27) and Eqs. (4.28), as one can explicitly check. Moreover it holds true in general (i.e. for any improved HF resummation) for scales such that $s^{2} \gg v^{2}$, since we have in this limit

$$
\tilde{\Omega}_{1}^{(2)}\left(q_{s}\right)=\frac{\frac{1}{6} \lambda_{0}}{1-\frac{1}{6}(N+8) \lambda_{0} \tilde{I}^{(2)}\left(q_{s}\right)}+O\left(m^{2} / s^{2}\right),
$$

where $I^{(2)}$ stands for anyone of $I_{L L}^{(2)}, I_{T L}^{(2)}$, and $I_{T T}^{(2)}$. The renormalization condition thus defines the bare-torenormalized relation

$$
\lambda=\frac{\lambda_{0}}{1-\frac{N+8}{6} \lambda_{0} \tilde{I}^{(2)}\left(q_{s}\right)}+O\left(m^{2} / s^{2}\right)
$$

which is the usual 1-loop RG-invariant relation up to $O\left(\mathrm{~m}^{2} / \mathrm{s}^{2}\right)$ terms. This shows that the direct coupling re- 
normalization condition is approximately scale invariant for high renormalization scales. To obtain complete scale invariance it is enough to slightly modify the bare-torenormalized parametrization by changing the renormalization condition in the following way

$$
\begin{aligned}
6 \tilde{\Omega}_{1}^{(2)}\left(q_{s}\right) & =6 \lambda_{0} F_{1}\left(\lambda_{0} \tilde{I}_{L L}^{(2)}\left(q_{s}\right), \lambda_{0} \tilde{I}_{T L}^{(2)}\left(q_{s}\right), \lambda_{0} \tilde{I}_{T T}^{(2)}\left(q_{s}\right) ; N\right) \\
& =6 \lambda F_{1}\left(\lambda \tilde{J}_{L L}^{(2)}\left(q_{s}\right), \lambda \tilde{J}_{T L}^{(2)}\left(q_{s}\right), \lambda \tilde{J}_{T T}^{(2)}\left(q_{s}\right) ; N\right) \\
& =\lambda+O\left(m^{2} / s^{2}\right)
\end{aligned}
$$

where

$$
\begin{gathered}
\tilde{J}_{L L}^{(2)}(p)=I_{L L}^{(2)}-\tilde{I}_{L L}^{(2)}\left(q_{s}\right) \mathbf{1}, \quad \tilde{J}_{T L}^{(2)}(p)=I_{T L}^{(2)}-\tilde{I}_{L L}^{(2)}\left(q_{s}\right) \mathbf{1}, \\
\tilde{J}_{T T}^{(2)}(p)=I_{T T}^{(2)}-\tilde{I}_{L L}^{(2)}\left(q_{s}\right) \mathbf{1}
\end{gathered}
$$

are the properly subtracted finite loops (notice that the subtraction term is always the purely longitudinal $\left.I_{L L}^{(2)}\left(q_{s}\right)\right)$. Then, using the parametrization of $F_{1}$ in terms of the $f_{i}(\alpha ; N)$, one can verify that the bare-torenormalized relation reads exactly

$$
\lambda=\frac{\lambda_{0}}{1-\frac{N+8}{6} \lambda_{0} \tilde{I}_{L L}^{(2)}\left(q_{s}\right)} .
$$

We can see that this has the correct 1-loop-RG improved behavior with a specific choice of the finite parts. Then all the free parameters take the following manifestly finite forms

$$
\begin{aligned}
\Omega_{1}^{(2)} & =\lambda F_{1}\left(\lambda J_{L L}^{(2)}, \lambda J_{T L}^{(2)}, \lambda J_{T T}^{(2)} ; N\right), \\
\theta_{1} & =\lambda F_{2}\left(\lambda J_{L L}^{(2)}, \lambda J_{T L}^{(2)}, \lambda J_{T T}^{(2)} ; N\right), \\
\Omega_{4}^{(2)} & =\lambda F_{4}\left(\lambda J_{L L}^{(2)}, \lambda J_{T L}^{(2)}, \lambda J_{T T}^{(2)} ; N\right), \\
\theta_{4} & =\lambda F_{4}\left(\lambda J_{L L}^{(2)}, \lambda J_{T L}^{(2)}, \lambda J_{T T}^{(2)} ; N\right) .
\end{aligned}
$$

Moreover, consistently with the renormalization condition in Eq. (4.29), we can define the running coupling constant by means of the following equation

$\lambda(p) F_{1}\left(\lambda(p) \tilde{J}_{L L}^{(2)}(p), \lambda(p) \tilde{J}_{T L}^{(2)}(p), \lambda(p) \tilde{J}_{T T}^{(2)}(p) ; N\right)=\tilde{\Omega}_{1}^{(2)}(p)$

whose solution is simply the extension of Eq. (4.30) to arbitrary momentum

$$
\lambda(p)=\frac{\lambda}{1-\frac{N+8}{6} \lambda \tilde{J}_{L L}^{(2)}(p)} .
$$

In terms of this and of the subtracted integrals we can write the renormalized form of the Fourier transform of $\alpha$ :

$$
\tilde{\alpha}(p)=\lambda(p)\left[\tilde{J}_{T L}^{(2)}(p)-\tilde{J}_{L L}^{(2)}(p)\right] .
$$

Notice that $\alpha$ becomes small for large (but smaller than the Landau pole) spatial momentum $q_{s}$. More precisely it is of order $O\left(\lambda\left(q_{s}\right) v^{2} / s^{2}\right)$. Because of the Landau pole, the cutoff of the theory cannot be removed but should be fixed to values suitably smaller than the pole (i.e. such that $\lambda\left(q_{\Lambda}\right)$ is of order one). The mass scale of the theory, that is $v$, is much smaller than the cutoff itself. Therefore for spatial momenta with values near the cutoff $\alpha$ is small. Then the perturbative (in $\alpha$ ) matching conditions assure that the different allowed choices of the free parameters give the same results to order $O\left(\lambda\left(q_{\Lambda}\right) v^{2} / \Lambda^{2}\right)$. In this sense we can say that all the class of improved HF approximations shares the same UV behavior.

One can easily check that if we renormalize at scale $s$ with coupling constant $\lambda$ and at scale $s^{\prime}$ with coupling constant $\lambda^{\prime}=\lambda\left(q_{s^{\prime}}\right)$ we define the same bare coupling constant. That is to say that the bare-to-renormalized relation in Eq. (4.30) is RG invariant. As a consequence the expressions of the parameters in terms of $\lambda_{0}$ and $I^{(2)}$ in Eqs. (4.23) can be thought as manifestly scale invariant definitions.

\section{CONCLUSIONS}

In this paper we extended to the case of spontaneously broken symmetry the improvement of the HF approach to the $O(N)$ scalar theory that was proposed in Ref. [33]. In contrast to the standard HF approximation, our improved one is renormalizable, RG invariant, and correctly gapless in the Goldstone sector. However, it is not unique, except for $N=1$, because the mass difference between the longitudinal sector and the transverse Goldstone sector allows for a richer structure of renormalization finite parts that cannot be fully restricted by the requirements of renormalizability and RG invariance. As a consequence, an entire class of improvements is identified, parametrized by the four functions $f_{A}(\alpha ; N)$ which must satisfy some further constraints explicitly written in the previous section. It is important to stress that, albeit of functional type, the remaining freedom is much smaller than what simple dimensional analysis would allow even when simple momentumindependent observables are concerned (the momentum dependences in our approximation are anyway fixed by construction to be of mean-field type). This makes it difficult, although not a priori impossible, to identify consistent schemes to further constrain the functions $f_{A}(\alpha ; N)$ by requiring agreement in suitable calculations beyond one loop. Work on this direction is in progress. 
[1] J. Berges, AIP Conf. Proc. 739, 3 (2004); J. Berges and J. Serreau, hep-ph/0410330; J. Berges and J. Serreau, hep$\mathrm{ph} / 0302210$.

[2] E. Manfredini, hep-ph/0101202.

[3] F. Cooper and E. Mottola, Phys. Rev. D 36, 3114 (1987); F. Cooper, S. Habib, Y. Kluger, E. Mottola, J. P. Paz, and P. R. Anderson, Phys. Rev. D 50, 2848 (1994).

[4] D. Boyanovsky, H. J. de Vega, R. Holman, D. S. Lee, and A. Singh, Phys. Rev. D 51, 4419 (1995).

[5] J. Baacke and K. Heitmann, Phys. Rev. D 62, 105022 (2000); J. Baacke, K. Heitmann, and C. Patzold, Phys. Rev. D 57, 6406 (1998).

[6] C. Destri and E. Manfredini, Phys. Rev. D 62, 025007 (2000).

[7] G. Amelino-Camelia and S. Y. Pi, Phys. Rev. D 47, 2356 (1993).

[8] C. Destri and E. Manfredini, Phys. Rev. D 62, 025008 (2000).

[9] S. Michalski, hep-ph/0301134.

[10] T. Matsui, hep-ph/0111277; Y. Tsue, D. Vautherin, and T. Matsui, Prog. Theor. Phys. 102, 313 (1999).

[11] O. J. P. Eboli, R. Jackiw, and S. Y. Pi, Phys. Rev. D 37, 3557 (1988).

[12] F. Cooper, S. Y. Pi, and P. N. Stancioff, Phys. Rev. D 34, 3831 (1986).

[13] J. Berges and J. Cox, Phys. Lett. B 517, 369 (2001).

[14] S. Habib, Y. Kluger, E. Mottola, and J. P. Paz, Phys. Rev. Lett. 76, 4660 (1996); F. Cooper, S. Habib, Y. Kluger, and E. Mottola, Phys. Rev. D 55, 6471 (1997); B. Mihaila, T. Athan, F. Cooper, J. Dawson, and S. Habib, Phys. Rev. D 62, 125015 (2000).

[15] D. Boyanovsky and H. J. de Vega, Phys. Rev. D 47, 2343 (1993); D. Boyanovsky, H.J. de Vega, R. Holman, and J. F. J. Salgado, Phys. Rev. D 54, 7570 (1996); D. Boyanovsky, C. Destri, H. J. de Vega, R. Holman, and J. Salgado, Phys. Rev. D 57, 7388 (1998).

[16] C. Wetterich, Phys. Rev. E 56, 2687 (1997); L. M. A. Bettencourt and C. Wetterich, Phys. Lett. B 430, 140 (1998); G. F. Bonini and C. Wetterich, Phys. Rev. D 60, 105026 (1999).

[17] M. Salle and J. Smit, Phys. Rev. D 67, 116006 (2003); M. Salle, J. Smit, and J. C. Vink, Phys. Rev. D 64, 025016 (2001); G. Aarts and J. Smit, Phys. Rev. D 61, 025002 (2000).

[18] J. Baacke and S. Michalski, Phys. Rev. D 65, 065019
(2002).

[19] E. Calzetta and B. L. Hu, Phys. Rev. D 37, 2878 (1988).

[20] J. M. Cornwall, R. Jackiw, and E. Tomboulis, Phys. Rev. D 10, 2428 (1974).

[21] J. Baacke and S. Michalski, hep-ph/0312031; J. Baacke and S. Michalski, Phys. Rev. D 67, 085006 (2003); J. Baacke and S. Michalski, hep-ph/0409153; J. Baacke and A. Heinen, Phys. Rev. D 69, 083523 (2004).

[22] D. Dominici and U. M. B. Marconi, Phys. Lett. B 319, 171 (1993).

[23] J. Berges and J. Serreau, Phys. Rev. Lett. 91, 111601 (2003); J. Berges and M. M. Muller, hep-ph/0209026; G. Aarts, D. Ahrensmeier, R. Baier, J. Berges, and J. Serreau, Phys. Rev. D 66, 045008 ( 2002); J. Berges, Nucl. Phys. A699, 847 ( 2002).

[24] F. Cooper, J. F. Dawson, and B. Mihaila, Phys. Rev. D 71, 096003 (2005).

[25] J. T. Lenaghan and D.H. Rischke, J. Phys. G 26, 431 (2000).

[26] S. Y. Pi and M. Samiullah, Phys. Rev. D 36, 3128 (1987).

[27] J. Baacke, K. Heitmann, and C. Patzold, Phys. Rev. D 55, 2320 (1997).

[28] F. Cooper, B. Mihaila, and J. F. Dawson, Phys. Rev. D 70, 105008 (2004).

[29] A. Jakovac and Z. Szep, Phys. Rev. D 71, 105001 (2005); A. Jakovac and Z. Szep, hep-ph/0408360.

[30] J. P. Blaizot, E. Iancu, and U. Reinosa, Phys. Lett. B 568, 160 (2003); J. P. Blaizot, E. Iancu, and U. Reinosa, Nucl. Phys. A736, 149 (2004).

[31] H. van Hees and J. Knoll, hep-ph/0210262.

[32] J. Berges, S. Borsanyi, U. Reinosa, and J. Serreau, Ann. Phys. (N.Y.) 320, 344 (2005).

[33] C. Destri and A. Sartirana, Phys. Rev. D 72, 065003 (2005); H. van Hees and J. Knoll, hep-ph/0202263.

[34] G. Baym and G. Grinstein, Phys. Rev. D 15, 2897 (1977).

[35] H. van Hees and J. Knoll, Phys. Rev. D 66, 025028 (2002); Y. B. Ivanov, F. Riek, and J. Knoll, Phys. Rev. D 71, 105016 (2005); Y. B. Ivanov, F. Riek, H. van Hees, and J. Knoll, Phys. Rev. D 72, 036008 ( 2005).

[36] F. Cooper, hep-th/9504073.

[37] K. c. Chou, Z. b. Su, B.1. Hao, and L. Yu, Phys. Rep. 118, 1 (1985).

[38] J. Baacke, K. Heitmann, and C. Patzold, Phys. Rev. D 57, 6398 (1998). 